\title{
First-principles constitutive equation for suspension rheology
}

\author{
J. M. Brader, ${ }^{1, *}$ M. E. Cates, ${ }^{2}$ and M. Fuchs ${ }^{1}$ \\ ${ }^{1}$ Fachbereich Physik, Universität Konstanz, D-78457 Konstanz, Germany \\ ${ }^{2}$ SUPA, School of Physics, The University of Edinburgh, Mayfield Road, Edinburgh EH9 3JZ, United Kingdom
}

(Received 9 May 2012; published 13 August 2012)

\begin{abstract}
We provide a detailed derivation of a recently developed first-principles approach to calculating averages in systems of interacting, spherical Brownian particles under time-dependent flow. Although we restrict ourselves to flows which are both homogeneous and incompressible, the time dependence and geometry (e.g., shear and extension) are arbitrary. The approximations formulated within mode-coupling theory are particularly suited to dense colloidal suspensions and capture the slow relaxation arising from particle interactions and the resulting glass transition to an amorphous solid. The delicate interplay between slow structural relaxation and timedependent external flow in colloidal suspensions thus may be studied within a fully tensorial theory.
\end{abstract}

DOI: 10.1103/PhysRevE.86.021403

PACS number(s): 82.70.Dd

\section{INTRODUCTION}

Imposing flow on a colloidal suspension distorts the microstructure away from that of the quiescent state and induces a nontrivial macroscopic stress response. The microscopic dynamics underlying this macroscopic behavior are governed by a combination of potential, Brownian, and hydrodynamic forces which interact in a complicated fashion with the solvent flow field, as described by the Smoluchowski equation $[1,2]$. The subtle balance between these various physical mechanisms gives rise to a rich phenomenology but also serves to complicate the formulation of tractable theoretical approaches [3]. In the present work we will present details of a first-principles theory, first outlined in Ref. [4], which provides a unified description of the mechanical response of colloidal liquids and glasses to external flow, albeit in the absence of hydrodynamic interactions.

Colloidal suspensions are of great importance for practical applications and the performance of many commercial products and industrial processes often depends on the rheological nonlinearities which occur when colloidal particles are added to a Newtonian solvent [5]. For example, the phenomena of shear thinning, shear thickening, and yielding are relevant for the even spreading of paints, shock absorption in automobiles and the flow of toothpaste, respectively. In order to control and tune the rheology of a suspension to meet the needs of a specific application, it is, thus, necessary to have an understanding of how the microscopic interactions between the constituents influence the macroscopic response [6]. The challenge to statistical physics is to identify tractable approximation schemes which capture the relevant physics while remaining simple enough for concrete calculations to be performed.

Quiescent monodisperse systems of spherical particles display an equilibrium phase diagram with colloidal gas, fluid, and crystalline phases, analogous to those found in simple fluids [7]. By introducing a sufficent degree of polydispersity, the mechanisms leading to crystallization can be suppressed and the system may undergo dynamical arrest to a metastable glass or gel phase. Experimentally, such arrested states are charac-

\footnotetext{
*Present address: Department of Physics, University of Fribourg, CH-1700 Fribourg, Switzerland.
}

terized by very slow structural relaxation [8-10]. As the system remains nonergodic on measureable time scales, it appears as a soft amorphous solid with a corresponding elastic modulus. Many features of the dynamical arrest observed in colloidal experiments are captured by the mode-coupling theory (MCT), both for repulsive glasses for which the arrest is caused by steric hinderance [11] and attractive glasses and gels where interparticle attraction dominates the dynamics [12,13] (although ageing dynamics are neglected within standard MCT).

It is fair to say that the MCT-based theoretical understanding of quiescent glasses is now in a fairly advanced state and that the linear response regime is under control. However, much less is known about the nonlinear response of arrested states and, despite progress, consensus remains to be achieved regarding the fundamental physical mechanisms at work. A key feature of systems under flow is the competition between the time scale of structural relaxation, which becomes very large close to the glass transition, and the time scale defined by the inverse of the flow rate. This competition is captured in an elegant way by generalized MCT treatments [14-17] for which the memory function generating the slow relaxation becomes reduced by shear via the mechanism of wave-vector advection. Various nonlinear rheological phenomena related to shear thinning are found to arise. The earliest generalizations of MCT to treat nonlinear rheology focused on the special case of shear flow [14,15] (see also Ref. [16] for a detailed account). More recently, the theory has been extended, first, to treat time-dependent shear $[17,18]$ and, subsequently, to treat arbitrary time-dependent flow [4]. These final developments have elevated the theory to the status of a full constitutive model in the sense that nonsteady three-dimensional flows can be addressed. Although full numerical solution of the equations in three dimensions has not yet been achieved, a simplified schematic version of the tensorial theory has been developed which provides sensible predictions regarding the flow and yielding behavior of systems with densities close to and above the glass transition [19].

We would like to clarify the relationship of the present work with our previous publications on this topic. The original outline for extending MCT to treat nonlinear rheology under steady shear flow was presented in Ref. [14]. Due to the technical difficulty of numerically solving the closed MCT equations in three dimensions, tractable schematic and 
semischematic models aiming to capture the essential physical mechanisms were developed in Ref. [20]. The approach of Ref. [14] was generalized to treat time-dependent shear flow and applied to the special case of shear step-strain [17]. During the subsequent development of the theory we made significant technical improvements which involved an alternative definition of the transient density correlator from that employed in Refs. [14,17,20]. [The improved definition is given by (68) below.] This new definition ensures that the initial decay rate [see (90) below] remains positive for all shear rates, which improves the numerical stability of the theory and leads to simpler and more elegant expressions. It is this improved formulation which was employed in the short paper [4] and which will be explained in detail in this work. The simple-shear theory recently presented in Ref. [16] also employed the improved formalism and is a special case of the results presented here. It has already been solved numerically for steady shear in two dimensions leading to results for stresses, structure, and single-particle motion in qualitative agreement with Brownian dynamics simulations [21,22].

In this paper we present full details of the tensorial timedependent theory that was outlined in Ref. [4]. Our theory starts from a well-defined microscopic starting point (the Smoluchowski equation) and leads directly to an approximation for the time-dependent nonequilibrium distribution function from which average quantities can be calculated. This includes, but is not limited to, an approximation for the macroscopic stress tensor as a functional of the velocity gradient tensor.

The paper is structured as follows: In Sec. II we define the microscopic dynamics of the colloidal particles. In Sec. III considerations of translational invariance are employed to identify the deformation measures appropriate for describing the system under flow. In Sec. IV we develop the integrationthrough-transients formalism which generates a generalized Green-Kubo relation for the macroscopic stress tensor, the mode-coupling approximation of which is outlined in Sec. V. Our approximate expression for the stress requires knowledge of the transient density correlator. In Sec. VI we develop a mode-coupling equation of motion for this quantity which captures the competition between slow structural relaxation and the fluidizing effect of flow. In Sec. VII we consider application of the integration-through-transients formalism to calculation of the distorted structure factor and show how an integration over the anisotropy, which encodes the microstructural distortion, recovers the constitutive equation as obtained earlier in Sec. V. Finally, in Sec. VIII we give a discussion and outlook to future work.

\section{MICROSCOPIC DYNAMICS}

We consider a system of $N$ spherical Brownian particles of diameter $d$ dispersed in a solvent with a specified time-dependent velocity profile $\mathbf{v}(\mathbf{r}, t)=\kappa(t) \cdot \mathbf{r}$, where the velocity gradient $\kappa(t)$ is taken to be a fixed input quantity. We assume incompressible flow, $\operatorname{Tr} \kappa(t)=0$, and will, from the outset, neglect hydrodynamic interactions. Our choice to neglect hydrodynamics is largely motivated by the desire to obtain a computationally tractable theory, but may also be physically jusifiable for high volume fraction states close to the glass transition, where particle motion is slow. Given these assumptions, the distribution function of particle positions obeys the simplified Smoluchowski equation [1]

$$
\frac{\partial \Psi(t)}{\partial t}=\Omega(t) \Psi(t),
$$

where the Smoluchowski operator is given by

$$
\Omega(t)=\sum_{i} \boldsymbol{\partial}_{i} \cdot\left[\boldsymbol{\partial}_{i}-\mathbf{F}_{i}-\kappa(t) \cdot \mathbf{r}_{i}\right] .
$$

In order to simplify notation we have set both the thermal energy $k_{B} T$ and bare diffusion coefficient $D_{0}$ equal to unity. The total force acting on particle $i$ due to potential interations is given by $\mathbf{F}_{i}=-\boldsymbol{\partial}_{i} U_{N}$, where $U_{N}$ is the total potential energy.

\section{TRANSLATIONAL INVARIANCE}

Employing a spatially constant velocity gradient has the consequence that the two-time correlation functions are invariant with respect to spatial translation. The requirement that translational invariance holds for the two-time correlations provides a method to identify the relevant affine deformation measures required for our subsequent analysis. A prerequisite is an understanding of the invariance properties of the timedependent distribution function $\Psi(t)$. If the system is taken to be in thermodynamic equilibrium at some initial time $t_{0}$ which, without loss of generality, can be taken as the time origin $t_{0}=0$, the Smoluchowski equation (1) may be formally solved to obtain the distribution at later times,

$$
\Psi(t)=e_{+}^{\int_{0}^{t} d s \Omega(s)} \Psi_{e}
$$

where we have introduced a time-ordered exponential function (Appendix A). In the following, we will, first, use the formal solution (3) to prove the translational invariance of $\Psi(t)$ and then use this result to analyze the two-time correlation functions.

\section{A. Distribution function}

The fact that $\kappa(t)$ does not depend on spatial coordinates suggests that the translational invarance of the equilibrium state will be preserved by the Smoluchowski dynamics; only relative particle motion is physically relevant. However, proof of this expectation for a general time-dependent flow is complicated by the fact that the Smoluchowski operator is itself not translationally invariant. Shifting all particle coordinates by a constant vector $\mathbf{r}_{i}^{\prime}=\mathbf{r}_{i}+\mathbf{a}$ leads to the shifted Smoluchowski operator

$$
\Omega\left(\Gamma^{\prime}, t\right)=\Omega(\Gamma, t)-\mathbf{a} \cdot \boldsymbol{\kappa}^{T}(t) \cdot \mathbf{P} \equiv \Omega(\Gamma, \mathbf{t})+\mathbf{A}(\mathbf{t}),
$$

where $\mathbf{P} \equiv \sum_{i} \boldsymbol{\partial}_{i}$ and we have used

$$
\mathbf{P} \cdot \boldsymbol{\kappa}(t) \cdot \mathbf{a}=\mathbf{a} \cdot \boldsymbol{\kappa}^{T}(t) \cdot \mathbf{P} .
$$

The positions of all particles are represented by $\Gamma \equiv$ $\left\{\mathbf{r}_{1}, \ldots, \mathbf{r}_{N}\right\}$. Substitution of (4) into (3) gives the distribution function following the shift

$$
\Psi\left(\Gamma^{\prime}, t\right)=e_{+}^{\int_{0}^{t} d s(\Omega(\Gamma, s)+A(s))} \Psi_{e}(\Gamma),
$$

where we have used the obvious translational invariance of the equilibrium distribution $\Psi_{e}\left(\Gamma^{\prime}\right)=\Psi_{e}(\Gamma)$. The next step is to 
write the time-ordered exponential explicitly in terms of its series definition

$$
\begin{aligned}
e_{+}^{\int_{0}^{t} d s(\Omega(s)+A(s))} \\
=1+\int_{t_{1}}^{t_{2}} d s_{1}\left[\Omega\left(s_{1}\right)+A\left(s_{1}\right)\right] \\
\quad+\int_{t_{1}}^{t_{2}} d s_{1} \int_{t_{1}}^{s_{1}} d s_{2}\left[\Omega\left(s_{1}\right)+A\left(s_{1}\right)\right]\left[\Omega\left(s_{2}\right)+A\left(s_{2}\right)\right] \\
\quad+\cdots .
\end{aligned}
$$

In order to simplify this expression we define the commutators

$$
\begin{aligned}
c^{(2)}\left(s_{1}, s_{2}\right) & \equiv\left[A\left(s_{1}\right), \Omega\left(s_{2}\right)\right] \\
c^{(3)}\left(s_{1}, s_{2}, s_{3}\right) & \equiv\left[c^{(2)}\left(s_{1}, s_{2}\right), \Omega\left(s_{3}\right)\right] \\
& \vdots \\
c^{(n)}\left(s_{1}, \ldots, s_{n}\right) & \equiv\left[c^{(n-1)}\left(s_{1}, \ldots, s_{n-1}\right), \Omega\left(s_{n}\right)\right] .
\end{aligned}
$$

Due to the fact $A(t)$ is independent of spatial coordinates, simple expressions are obtained for the commutators

$$
\begin{aligned}
c^{(2)}\left(s_{1}, s_{2}\right) & =\mathbf{a} \cdot \boldsymbol{\kappa}^{T}\left(s_{1}\right) \boldsymbol{\kappa}^{T}\left(s_{2}\right) \cdot \mathbf{P} \\
c^{(3)}\left(s_{1}, s_{2}, s_{3}\right) & =-\mathbf{a} \cdot \boldsymbol{\kappa}^{T}\left(s_{1}\right) \boldsymbol{\kappa}^{T}\left(s_{2}\right) \boldsymbol{\kappa}^{T}\left(s_{3}\right) \cdot \mathbf{P} \\
& \vdots \\
c^{(n)}\left(s_{1}, \cdots, s_{n}\right) & =(-1)^{n} \mathbf{a} \cdot \prod_{i=1}^{n} \boldsymbol{\kappa}^{T}\left(s_{i}\right) \cdot \mathbf{P} .
\end{aligned}
$$

Newton's third law states that the sum of internal forces in the system must be zero. It follows that

$$
\mathbf{P} \psi_{e}=\sum_{i} \boldsymbol{\partial}_{i} \frac{e^{-U_{N}}}{Z}=\psi_{e} \sum_{i} \mathbf{F}_{i}=0,
$$

where $Z$ is the canonical partition function. We thus have

$$
\begin{gathered}
A\left(s_{1}\right) \Psi_{e}(\Gamma)=0 \\
c^{(n)}\left(s_{1}, \ldots, s_{n}\right) \Psi_{e}(\Gamma)=0 .
\end{gathered}
$$

For each term in (7) the commutation relations (8) may be used to bring all factors of $\Omega$ to the left of the integrand. Application of the relations (11) and (12) then eliminates all terms involving $\boldsymbol{\kappa}$. We are, thus, left with

$$
\begin{aligned}
\Psi\left(\Gamma^{\prime}, t\right)= & {\left[1+\int_{t_{1}}^{t_{2}} d s_{1} \Omega\left(\Gamma, s_{1}\right)\right.} \\
& \left.+\int_{t_{1}}^{t_{2}} d s_{1} \int_{t_{1}}^{s_{1}} d s_{2} \Omega\left(\Gamma, s_{1}\right) \Omega\left(\Gamma, s_{2}\right)+\cdots\right] \Psi_{e} \\
= & \exp _{+}\left[\int_{0}^{t} d s \Omega(\Gamma, s)\right] \Psi_{e}=\Psi(\Gamma, t)
\end{aligned}
$$

which is the desired result. We have, thus, demonstrated that the distibution function is translationally invariant for any homogeneous flow field $\kappa(t)$ (although it will be anisotropic, reflecting the symmetry of the imposed velocity gradient).

\section{B. Two-time correlation function}

We now consider the properties under uniform translation of the two-time correlation functions, namely the correlation of two wave-vector-dependent fluctuations. Without loss of generality we may express the wave-vector-dependent fluctuations as

$$
f_{\mathbf{q}}\left(\Gamma, t, t^{\prime}\right)=e_{-}^{\int_{t^{\prime}}^{t} d s \Omega^{\dagger}(\Gamma, s)} \sum_{i} X_{i}^{f}(\Gamma) e^{i \mathbf{q} \cdot \mathbf{r}_{i}},
$$

where the variable $X_{i}^{f}$ depends on the fluctuation under consideration. The adjoint Smoluchowski operator can be obtained from (1) by partial integration

$$
\Omega^{\dagger}(t)=\sum_{i}\left[\partial_{i}+\mathbf{F}_{i}+\mathbf{r}_{i} \cdot \boldsymbol{\kappa}^{T}(t)\right] \cdot \boldsymbol{\partial}_{i} .
$$

In contrast to the distribution function, which evolves from time $t^{\prime}$ to time $t$ according to the propagator $\exp _{+} \int_{t^{\prime}}^{t} d s \Omega(s)$, the time evolution of fluctuations ("observables") is dictated by the adjoint propagator $\exp _{-} \int_{t^{\prime}}^{t} d s \Omega^{\dagger}(s)$, where the operation of taking the adjoint reverses the time ordering [see Eqs. (A9) and (A10)]. In the present work, we will consider only fluctuations for which $X_{i}^{f}(\Gamma)$ is translationally invariant. This assumption holds, for example, in the case of the density $\rho_{\mathbf{q}}(t)$, obtained by setting $X_{i}^{\rho}=1$ in (15) and the less familiar case of wave-vector-dependent stress fluctuations $\sigma_{\alpha \beta}\left(\mathbf{q},\left\{\mathbf{r}_{i}\right\}\right)[23,24]$, obtained by setting

$$
\left(X_{i}^{\sigma}\right)_{\alpha \beta}=\delta_{\alpha \beta}-\frac{1}{2} \sum_{j \neq i} \frac{r_{i j}^{\alpha} r_{i j}^{\beta}}{r_{i j}} \frac{d u\left(r_{i j}\right)}{d r_{i j}}
$$

in (15), where $u(r)$ is the pair interaction potential. According to this definition $\sigma_{\alpha \beta}\left(\mathbf{q},\left\{\mathbf{r}_{i}\right\}\right)$ has dimensions of energy (recall that we set $k_{B} T=1$ ) and thus requires division by a volume to become a true stress. One should thus bear in mind that the familiar macroscopic stress arises from taking the average of $\sigma_{\alpha \beta}\left(\mathbf{q}=0,\left\{\mathbf{r}_{i}\right\}\right) / V$.

The two-time correlation function is formally defined by

$$
C_{f_{\mathbf{q}} g_{\mathbf{k}}}\left(t, t^{\prime}\right)=\int d \Gamma \Psi\left(t^{\prime}\right) f_{\mathbf{q}}^{*} e_{-}^{\int_{t^{\prime}}^{t} d s \Omega^{\dagger}(s)} g_{\mathbf{k}} .
$$

As in the previous subsection, we now shift all particle coordinates by a constant vector, $\mathbf{r}_{i} \rightarrow \mathbf{r}_{i}+\mathbf{a}$ to obtain the shifted adjoint Smoluchowski operator

$$
\Omega^{\dagger}\left(\Gamma^{\prime}, t\right)=\Omega^{\dagger}(\Gamma, t)+\mathbf{a} \cdot \boldsymbol{\kappa}^{T}(t) \cdot \mathbf{P} \equiv \Omega^{\dagger}(\Gamma, t)+A^{\dagger}(t) .
$$

The distribution function appearing in (18) is invariant with respect to this shift as proven in (14). To make further progress we employ the identity (B1) to factorize the ordered exponential

$$
\begin{aligned}
e_{-}^{\int_{t^{\prime}}^{t} d s} \Omega^{\dagger}(\Gamma, s)+A^{\dagger}(s) \\
=e_{-}^{\int_{t^{\prime}}^{t} d s \Omega^{\dagger}(\Gamma, s)} \\
\quad \times \exp _{-}\left[\int_{t^{\prime}}^{t} d s e_{+}^{-\int_{s}^{t} d s^{\prime} \Omega^{\dagger}\left(\Gamma, s^{\prime}\right)} A^{\dagger}(s) e_{-}^{\int_{s}^{t} d s^{\prime} \Omega^{\dagger}\left(\Gamma, s^{\prime}\right)}\right] .
\end{aligned}
$$

The argument of the second exponential is a complicated "interaction representation" of the operator $A^{\dagger}(s)$ but can be simplified considerably using the nested commutator expansion (C2) derived in Appendix C. Defining the 
commutators

$$
\begin{aligned}
b^{(2)}\left(s_{1}, s_{2}\right) & \equiv\left[\Omega^{\dagger}\left(s_{1}\right), A^{\dagger}\left(s_{2}\right)\right] \\
b^{(3)}\left(s_{1}, s_{2}, s_{3}\right) & \equiv\left[\Omega^{\dagger}\left(s_{1}\right),\left[\Omega^{\dagger}\left(s_{2}\right), A^{\dagger}\left(s_{3}\right)\right]\right] \\
b^{(4)}\left(s_{1}, s_{2}, s_{3}, s_{4}\right) & \equiv\left[\Omega^{\dagger}\left(s_{1}\right),\left[\Omega^{\dagger}\left(s_{2}\right),\left[\Omega^{\dagger}\left(s_{3}\right), A^{\dagger}\left(s_{4}\right)\right]\right]\right],
\end{aligned}
$$

we find that

$$
\begin{aligned}
b^{(2)}\left(s_{1}, s_{2}\right) & =-\mathbf{a} \cdot \boldsymbol{\kappa}^{T}\left(s_{2}\right) \boldsymbol{\kappa}^{T}\left(s_{1}\right) \cdot \mathbf{P} \\
b^{(3)}\left(s_{1}, s_{2}, s_{3}\right) & =\mathbf{a} \cdot \boldsymbol{\kappa}^{T}\left(s_{3}\right) \boldsymbol{\kappa}^{T}\left(s_{2}\right) \boldsymbol{\kappa}^{T}\left(s_{1}\right) \cdot \mathbf{P} \\
& \vdots \\
b^{(n)}\left(s_{1}, \cdots, s_{n}\right) & =(-1)^{(n+1)} \mathbf{a} \cdot \prod_{i=0}^{n-1} \boldsymbol{\kappa}^{T}\left(s_{n-i}\right) \cdot \mathbf{P} .
\end{aligned}
$$

Using (21) and (22) in the expansion (C2) yields a simplification of the interaction representation operator

$$
\begin{aligned}
e_{+}^{-\int_{s}^{t} d s^{\prime} \Omega^{\dagger}\left(\Gamma, s^{\prime}\right)} A^{\dagger}(s) e_{-}^{\int_{s}^{t} d s^{\prime} \Omega^{\dagger}\left(\Gamma, s^{\prime}\right)} \\
=\mathbf{a} \cdot \boldsymbol{\kappa}^{T}(s)\left[\mathbf{1}+\int_{s}^{t} d s_{1} \boldsymbol{\kappa}^{T}\left(s_{1}\right)\right. \\
\left.\quad+\int_{s}^{t} d s_{1} \int_{s}^{s_{1}} d s_{2} \boldsymbol{\kappa}^{T}\left(s_{2}\right) \boldsymbol{\kappa}^{T}\left(s_{1}\right)+\cdots\right] \cdot \mathbf{P} \\
=-\mathbf{a} \cdot\left[\frac{\partial}{\partial s} e_{-}^{\int_{s}^{t} d s^{\prime} \boldsymbol{\kappa}^{T}\left(s^{\prime}\right)}\right] \cdot \mathbf{P},
\end{aligned}
$$

where the last equality follows from (A8) and the definition of the ordered exponential (A2). Substitution into (20) yields the useful result

$$
\begin{aligned}
& e_{-}^{\int_{t^{\prime}}^{t} d s \Omega^{\dagger}(\Gamma, s)+A^{\dagger}(s)} \\
& \quad=e_{-}^{\int_{t^{\prime}}^{t} d s \Omega^{\dagger}(\Gamma, s)} \exp \left[\mathbf{a} \cdot\left(-\mathbf{1}+e_{-}^{\int_{t^{\prime}}^{t} d s \kappa^{T}(s)}\right) \cdot \mathbf{P}\right],
\end{aligned}
$$

Substitution of (24) into (18), noting that $\mathbf{P} g_{\mathbf{k}}=i \mathbf{k} g_{\mathbf{k}}$, and using the adjoint relation (A10) leads to our final result,

$$
C_{f_{\mathbf{q}} g_{\mathbf{k}}}\left(t, t^{\prime}\right)=e^{-i\left(\mathbf{q}-\mathbf{k}\left(t, t^{\prime}\right)\right) \cdot \mathbf{a}} C_{f_{\mathbf{q}} g_{\mathbf{k}}}\left(t, t^{\prime}\right)
$$

where the time-dependent wave vector is given by

$$
\mathbf{k}\left(t, t^{\prime}\right)=\mathbf{k} \cdot e_{+}^{\int_{t^{\prime}}^{t} d s \kappa(s)}
$$

The condition of translational invariance thus requires that the phase factor vanishes and leads us the conclusion that fluctuation at wave vector $\mathbf{k}$ at earlier time $t^{\prime}$ is correlated with one at $\mathbf{q}=\mathbf{k}\left(t, t^{\prime}\right)$ at later time $t$, as a result of the affine flow. Equivalently, one can employ the inverse transformation (see Appendix A) to show that an earlier fluctuation at wave vector $\mathbf{k}=\overline{\mathbf{q}}\left(t, t^{\prime}\right)$ is correlated with a later one at wave vector $\mathbf{q}$, where

$$
\overline{\mathbf{q}}\left(t, t^{\prime}\right)=\mathbf{q} \cdot e_{-}^{-\int_{t^{\prime}}^{t} d s \kappa(s)} .
$$

In the following we will refer to (27) as the forward-advected wave vector and (26) as the reverse-advected wave vector.

\section{Rheological tensors and advection}

The ordered exponential identified in (27) may appear unfamiliar but is nothing more than the inverse of the deformation gradient tensor

$$
\boldsymbol{E}\left(t, t^{\prime}\right)=\frac{\partial \mathbf{r}(t)}{\partial \mathbf{r}\left(t^{\prime}\right)},
$$

a standard quantity in elasticity theory $[25,26]$. The deformation gradient transforms a vector ("material line") at time $t^{\prime}$ to a new vector at later time $t$. Thus, for spatially homogeneous deformations, $\mathbf{r}(t)$ is transformed from the past to the present via

$$
\mathbf{r}(t)=\boldsymbol{E}\left(t, t^{\prime}\right) \cdot \mathbf{r}\left(t^{\prime}\right),
$$

where $E_{\alpha \beta}=\partial r_{\alpha} / \partial r_{\beta}$, and from the present to the past using the inverse deformation gradient

$$
\mathbf{r}\left(t^{\prime}\right)=\boldsymbol{E}^{-1}\left(t, t^{\prime}\right) \cdot \mathbf{r}(t) .
$$

Taking the time derivative of (28) and applying the chain rule to the right-hand side generates a differential equation connecting the deformation to the velocity gradient

$$
\frac{\partial}{\partial t} \boldsymbol{E}\left(t, t^{\prime}\right)=\boldsymbol{\kappa}(t) \boldsymbol{E}\left(t, t^{\prime}\right) .
$$

Given the boundary condition $E(t, t)=1$ the general solution of (31) is, thus,

$$
\boldsymbol{E}\left(t, t^{\prime}\right)=e_{+}^{\int_{t^{\prime}}^{t} d s \kappa(s)},
$$

with inverse (see Appendix A)

$$
\boldsymbol{E}^{-1}\left(t, t^{\prime}\right)=e_{-}^{-\int_{t^{\prime}}^{t} d s \kappa(s)}
$$

The tensors (32) and (33) are precisely those identified from our consideration of the translational invariance of wave-vector-dependent density fluctuations, namely $\overline{\mathbf{k}}\left(t, t^{\prime}\right)=$ $\mathbf{k} \cdot \boldsymbol{E}^{-1}\left(t, t^{\prime}\right)$ and $\mathbf{k}\left(t, t^{\prime}\right)=\mathbf{k} \cdot \boldsymbol{E}\left(t, t^{\prime}\right)$.

\section{INTEGRATION THROUGH TRANSIENTS}

\section{A. Nonequilibrium distribution function}

If we now assume that the system was in thermodynamic equilibrium in the infinite past the formal solution (3) becomes

$$
\Psi(t)=e_{+}^{\int_{-\infty}^{t} d s \Omega(s)} \Psi_{e} .
$$

Despite being formally exact, the fact that the time evolution is entirely contained within the distribution function proves inconvenient when attempting to make a closure approximation. For this reason, we seek an alternative, but equivalent, solution for which the observables evolve in time and the distribution function remains fixed. The procedure is analogous to the passage from Schrödinger to Heisenberg pictures in quantum mechanics and will lead to a Dysonequation like representation of the transient dynamics [27].

Without loss of generality we can separate both the Smoluchowski operator and the distribution function into equilibrium and nonequilibrium contributions,

$$
\begin{aligned}
& \Omega(t)=\Omega_{e}+\delta \Omega(t), \\
& \Psi(t)=\Psi_{e}+\delta \Psi(t),
\end{aligned}
$$

where $\delta \Omega(t)=-\sum_{i} \partial_{i} \cdot\left[\kappa(t) \cdot \mathbf{r}_{i}\right]$ and $\delta \Psi(t)$ remains to be determined. The equilibrium distribution satisfies $\Omega_{e} \Psi_{e}=$ 0 , whereas nonequilibrium steady states are determined by 
$\Omega \Psi=0$. When acting on the equilibrium distribution the nonequilibrium Smoluchowski operator generates a term proportional to the stress tensor

$$
\Omega(t) \Psi_{e}=\delta \Omega(t) \Psi_{e}=[\kappa(t): \hat{\sigma}] \Psi_{e},
$$

where $\mathbf{A}: \mathbf{B} \equiv \sum_{\alpha \beta} A_{\alpha \beta} B_{\beta \alpha}$ and $\hat{\boldsymbol{\sigma}}$ is the zero wave-vector limit of the potential part of the stress tensor, which we now write in the following form:

$$
\hat{\sigma}_{\alpha \beta}=\delta_{\alpha \beta}-\sum_{i} r_{i}^{\alpha} F_{i}^{\beta} .
$$

In order to avoid any confusion, we note that a simple transformation to relative coordinates [28] suffices to demonstrate the equivalence of (38) to the more familiar Kirkwood form (17) [24]. Thus, despite first impressions, the compact expression (38) to be employed in our calculations does not depend on the choice of coordinate origin.

For the incompressible flow under consideration we have $\kappa(t): \delta=\operatorname{Tr} \kappa(t)=0$, such that the Kronecker $\delta$ in (38) is irrelevant at this stage of the calculation. Substitution of (35) and (36) into (1) thus yields an equation for $\delta \Psi(t)$,

$$
\frac{\partial}{\partial t} \delta \Psi(t)=\Omega(t) \delta \Psi(t)+[\kappa(t): \hat{\sigma}] \Psi_{e},
$$

which is a simple first-order differential equation with inhomogeneity $[\kappa(t): \hat{\sigma}] \Psi_{e}$. Assuming an equilibrium distribution in the infinite past, (39) is solved by

$$
\delta \Psi(t)=\int_{-\infty}^{t} d t_{1} e_{+}^{\int_{t_{1}}^{t} d s \Omega(s)}\left[\kappa\left(t_{1}\right): \hat{\boldsymbol{\sigma}}\right] \Psi_{e},
$$

as can be verified by either variation of parameters or the method of Greens functions. The full nonequilibrium distribution function is given by substitution of (40) into (36). The nonequilibrium average of an arbitrary function of the particle coordinates $f\left(\left\{\mathbf{r}^{N}\right\}\right)$, which need not be a scalar quantity, is, thus, given by

$$
\langle f\rangle^{\mathrm{ne}}=\langle f\rangle+\int d \Gamma \int_{-\infty}^{t} d t_{1} f e_{+}^{\int_{t_{1}}^{t} d s \Omega(s)}\left[\kappa\left(t_{1}\right): \hat{\boldsymbol{\sigma}}\right] \Psi_{e},
$$

where the integral over $\Gamma$ is a phase-space integral over all particle coordinates and $\langle\cdot\rangle$ denotes an equilibrium average. The final result, central to the present theoretical development, is obtained by partial integration

$$
\langle f\rangle^{\mathrm{ne}}=\langle f\rangle+\int_{-\infty}^{t} d t_{1}\left\langle\boldsymbol{\kappa}\left(t_{1}\right): \hat{\boldsymbol{\sigma}} e_{-}^{\int_{t_{1}}^{t} d s \Omega^{\dagger}(s)} f\right\rangle .
$$

The time development of the observable $f$ is, thus, generated by the adjoint operator.

As the test function $f$ is arbitrary, it may be removed to provide a formal operator expression for the distribution function

$$
\Psi(t)=\Psi_{e}+\int_{-\infty}^{t} d t_{1} \Psi_{e} \kappa\left(t_{1}\right): \hat{\boldsymbol{\sigma}} e_{-}^{\int_{t_{1}}^{t} d s \Omega^{\dagger}(s)} .
$$

This expression is equivalent to (34) and implies that the function to be averaged should be multiplied by (43) from the left and integrated over the particle coordinates. Equation (43) is the central result of the integration through transients formalism and we will demonstrate that this provides a very convenient starting point for analysis of the nonequilibrium dynamics of colloidal suspensions. We note that analogous expressions for the nonequilibrium distribution function have been considered by Evans and Morriss [27] and Chong and Kim [29], based on the thermostatted SLLOD equations of motion. A fundamental advantage of (43) over (34) is that it enables nonequilibrium averages to be expressed in terms of equilibrium averages, under the sole assumption that the system was in equilibrium in the infinite past. This leads to a Dyson-like representation of the dynamics, reminiscent of time-dependent perturbation theory in quantum mechanics. Within our formalism a range of nonergodic quiescent states can be generated from this initial state by imposing various flow histories. This includes the typical experimental protocol for which strong preshear is applied in order to erase memory and obtain a reproducible initial state from which the system age may be measured.

\section{B. Exact projection}

A potential pitfall of applying (43) arises from the existence of conservation laws which lead to zero eigenvalues of $\Omega^{\dagger}$ and which could, in principle, prevent convergence of the time integral. In the following we will construct an exact reformulation of (42) in which slow fluctuations are explicitly projected out, ensuring convergence of the integral for all values of $\mathbf{q}$ and, thus, preparing the ground for subsequent approximations.

For the Brownian dynamics under consideration only the particle number is conserved, $\partial_{t} \rho_{\mathbf{q}=0}=\Omega^{\dagger}(t) \rho_{\mathbf{q}=0}=0$, which suggests a possible divergence in the hydrodynamic limit when the arbitrary test function in (42) is chosen to be a density fluctuation, $f=\rho_{\mathbf{q}}$. Fortunately, this poses no difficulty, as the average in the integrand vanishes for all wave vectors,

$$
\left\langle\kappa(t): \hat{\sigma} e_{-}^{\int_{t_{1}}^{t} d s \Omega^{\dagger}(s)} \rho_{\mathbf{q}}\right\rangle=0
$$

which ensures that density fluctuations do not couple linearly to the nonequilibrium part of the distribution function. The fact that (44) holds at $q=0$ follows directly from inserting $\rho_{\mathbf{q}=0}=N$,

$$
\left\langle\boldsymbol{\kappa}(t): \hat{\boldsymbol{\sigma}} e_{-}^{\int_{t_{1}}^{t} d s \Omega^{\dagger}(s)} N\right\rangle=N\langle\boldsymbol{\kappa}(t): \hat{\boldsymbol{\sigma}}\rangle=0,
$$

where the second equality relies on the condition $\operatorname{Tr} \kappa(t)=0$. For nonzero $q$ the vanishing of the average in (44) is a consequence of translational invariance. Shifting all coordinates by a constant vector $\mathbf{r}_{i}^{\prime}=\mathbf{r}_{i}+\mathbf{a}$ and applying (24) leads to

$$
\begin{aligned}
\left\langle\boldsymbol{\kappa}(t): \hat{\boldsymbol{\sigma}} e_{-}^{\int_{t_{1}}^{t} d s \Omega^{\dagger}\left(\Gamma^{\prime}, s\right)} \rho_{\mathbf{q}}\right\rangle= & \exp \left[-i \mathbf{q} \cdot e_{-}^{-\int_{t^{\prime}}^{t} d s \boldsymbol{\kappa}(s)}\right] \\
& \times\left\langle\boldsymbol{\kappa}(t): \hat{\boldsymbol{\sigma}} e_{-}^{\int_{t_{1}}^{t} d s \Omega^{\dagger}(\Gamma, s)} \rho_{\mathbf{q}}\right\rangle .
\end{aligned}
$$

To prevent violation of translational invariance the average must be identically zero. Equation (44) is, thus, proven.

We now introduce the density projector $P$ and complement $Q$

$$
P=\sum_{\mathbf{k}}\left|\rho_{\mathbf{q}}\right\rangle \frac{1}{N S_{q}}\left\langle\rho_{\mathbf{q}}^{*}\right|, \quad Q=1-P
$$


where $S_{q}$ is the static structure factor $S_{q}=\left\langle\rho_{\mathbf{q}}^{*} \rho_{\mathbf{q}}\right\rangle / N$. In (47) we have introduced a bra-ket notation for wave-vectordependent fluctuations. The scalar product of two arbitrary variables is defined by an equilibrium average

$$
\left\langle f_{\mathbf{q}}^{*} \mid g_{\mathbf{q}}\right\rangle \equiv \int d \Gamma \Psi_{e} f_{\mathbf{q}}^{*}(\Gamma) g_{\mathbf{q}}(\Gamma) .
$$

The projectors (47) are both idempotent and orthogonal

$$
P^{2}=P, \quad Q^{2}=Q, \quad Q P=0,
$$

and, by construction, satisfy

$$
P\left|\rho_{\mathbf{q}}\right\rangle=\left|\rho_{\mathbf{q}}\right\rangle \quad Q\left|\rho_{\mathbf{q}}\right\rangle=0 .
$$

A direct consequence of (44) is that our central result (42) may be exactly rewritten as

$$
\langle f\rangle^{\mathrm{ne}}=\langle f\rangle+\int_{-\infty}^{t} d t_{1}\left\langle\kappa\left(t_{1}\right): \hat{\sigma} Q e_{-}^{\int_{t_{1}}^{t} d s \Omega^{\dagger}(s)} Q f\right\rangle,
$$

where we have again used $\langle\boldsymbol{\kappa}(t): \hat{\boldsymbol{\sigma}}\rangle=0$. To proceed further we consider a time-dependent generalization of a standard operator identity (see, e.g., Ref. [30] for the standard result)

$$
\begin{aligned}
e_{-}^{\int_{t_{1}}^{t} d s \Omega^{\dagger}(s)}= & e_{-}^{\int_{t_{1}}^{t} d s Q \Omega^{\dagger}(s)}+\int_{t_{1}}^{t} d s^{\prime} e_{-}^{\int_{t_{1}}^{s^{\prime}} d s \Omega^{\dagger}(s)} P \Omega^{\dagger}\left(s^{\prime}\right) \\
& \times e_{-}^{\int_{s^{\prime}}^{t} d s Q \Omega^{\dagger}(s)},
\end{aligned}
$$

which can easily be verified by differentiation (applying the rules from Appendix A). Substitution of this identity into (51) and using (44) eliminates the complicated second term in (52). Finally, exploiting the idempotency of $Q$ enables us to write

$$
e_{-}^{\int_{t_{1}}^{t} d s Q \Omega^{\dagger}(s)} Q=e_{-}^{\int_{t_{1}}^{t} d s Q \Omega^{\dagger}(s) Q} Q,
$$

which leads to our final result,

$$
\langle f\rangle^{\mathrm{ne}}=\langle f\rangle+\int_{-\infty}^{t} d t_{1}\left\langle\boldsymbol{\kappa}\left(t_{1}\right): \hat{\boldsymbol{\sigma}} Q e_{-}^{\int_{t_{1}}^{t} d s Q \Omega^{\dagger}(s) Q} Q f\right\rangle .
$$

The projected expression (54) is formally equivalent to (42). The advantage of this reformulation is that the absence of linear coupling to density fluctuations is explicit in the projected time evolution operator, which ensures that approximations to the equilibrium average in (42) will not introduce spurious linear couplings. It should be noted that these considerations rely on the incompressibility condition $\operatorname{Tr} \kappa=0$, the relaxation of which would indeed lead to a finite linear coupling for some compressible flows.

\section{CONSTITUTIVE EQUATION}

\section{A. Generalized Green-Kubo formula}

In order to address the rheology of colloidal suspensions, we now consider application of (43) to calculation of the macroscopic stress tensor. Replacing $f$ in (42) by the fluctuating stress tensor elements (38) yields a formally exact constitutive equation,

$$
\boldsymbol{\sigma}(t)=\frac{1}{V} \int_{-\infty}^{t} d t_{1}\left\langle\boldsymbol{\kappa}\left(t_{1}\right): \hat{\boldsymbol{\sigma}} e_{-}^{\int_{t_{1}}^{t} d s \Omega^{\dagger}(s)} \hat{\boldsymbol{\sigma}}\right\rangle .
$$

The stress tensor is, thus, a nonlinear functional of the velocity gradient tensor as $\kappa(t)$ appears in the adjoint Smoluchowski operator. Equation (55) thus goes beyond the standard GreenKubo formulas of linear response theory and makes possible a theoretical study of nonlinear rheology.

The general result (55) shows that for arbitrary timedependent flow the integrand must depend on two time arguments rather than a simple time difference. To illustrate this point, consider the special case of simple shear flow $\left(\kappa_{\alpha \beta}(t)=\dot{\gamma}(t) \delta_{x \alpha} \delta_{y \beta}\right.$, where $\dot{\gamma}(t)$ is the shear rate). The shear stress is given by

$$
\begin{aligned}
\sigma_{x y}(t) & =\int_{-\infty}^{t} d t_{1} \dot{\gamma}\left(t_{1}\right)\left[\frac{1}{V}\left\langle\hat{\sigma}_{x y} e_{-}^{\int_{t_{1}}^{t} d s \Omega^{\dagger}(s)} \hat{\sigma}_{x y}\right\rangle\right] \\
& \equiv \int_{-\infty}^{t} d t_{1} \dot{\gamma}\left(t_{1}\right) G\left(t, t_{1} ;[\kappa]\right),
\end{aligned}
$$

where the second equality serves to define the nonlinear shear modulus, which is a functional of the velocity gradient tensor. The lack of time translational invariance demonstrated by $G\left(t, t^{\prime} ;[\kappa]\right)$ is a general feature of two-time correlation functions in systems under time-dependent shear and is unavoidable if one wishes to go beyond linear response [31]. Time translational invariance can be recovered only by replacing in (56) the full evolution operator $\Omega^{\dagger}(t)$ with the time-independent equilibrium operator. For notational convenience, we will henceforth employ $G\left(t, t^{\prime}\right) \equiv G\left(t, t^{\prime} ;[\kappa]\right)$, omitting explicit reference to the functional dependence of the modulus on the velocity gradient.

\section{B. Mode-coupling approximation}

The exact generalized Green-Kubo expression for the stress tensor (55) requires approximation before explicit calculations can be performed. We begin by exactly re-expressing (55) in terms of the projected dynamics (54)

$$
\boldsymbol{\sigma}(t)=\frac{1}{V} \int_{-\infty}^{t} d t_{1}\left\langle\boldsymbol{\kappa}\left(t_{1}\right): \hat{\boldsymbol{\sigma}} Q e_{-}^{\int_{t_{1}}^{t} d s} Q \Omega^{\dagger}(s) Q \quad Q \hat{\boldsymbol{\sigma}}\right\rangle,
$$

which will be approximated by considering the overlap of stress fluctuations with the simplest relevant slow fluctuations. Due to the projector $Q$ in (57) the lowest nonzero order in fluctuation amplitude is taken to be $\rho_{\mathbf{k}} \rho_{\mathbf{p}}$, where $\mathbf{k}$ and $\mathbf{p}$ are two distinct wave vectors. We thus define a projection operator onto density pairs,

$$
P_{2}=\sum_{\mathbf{k}>\mathbf{p}}\left|\rho_{\mathbf{k}} \rho_{\mathbf{p}}\right\rangle \frac{1}{N^{2} S_{k} S_{p}}\left\langle\rho_{\mathbf{k}}^{*} \rho_{\mathbf{p}}^{*}\right|,
$$

which we assume will capture the dominant slow fluctuations. It should be pointed out that this projection operator is only approximate, as we have employed the Gaussian ansatz

$$
\left\langle\rho_{\mathbf{k}}^{*} \rho_{\mathbf{p}}^{*} \rho_{\mathbf{k}} \rho_{\mathbf{p}}\right\rangle \approx\left\langle\rho_{\mathbf{k}}^{*} \rho_{\mathbf{k}}\right\rangle\left\langle\rho_{\mathbf{p}}^{*} \rho_{\mathbf{p}}\right\rangle=N^{2} S_{k} S_{p}
$$

in the denominator [32]. Although the approximate projector (58) is not perfectly idempotent, it has the advantage that the normalization is expressed in terms of easily calculable equilibrium structure factors. Projecting onto density pairs 
yields an approximation to the stress

$$
\begin{aligned}
\boldsymbol{\sigma}(t)= & \frac{1}{V} \int_{-\infty}^{t} d t_{1}\left\langle\boldsymbol{\kappa}\left(t_{1}\right): \hat{\boldsymbol{\sigma}} Q P_{2} e_{-}^{\int_{t_{1}}^{t} d s Q \Omega^{\dagger}(s) Q} P_{2} Q \hat{\boldsymbol{\sigma}}\right\rangle \\
& =\sum_{\substack{\mathbf{k}>\mathbf{p} \\
\mathbf{k}^{\prime}>\mathbf{p}^{\prime}}} \frac{1}{V N^{4}} \int_{-\infty}^{t} d t_{1} \frac{V_{\mathbf{k}^{\prime} \mathbf{p}^{\prime}}^{(1)} V_{\mathbf{k p}}^{(2)}}{S_{k^{\prime}} S_{p^{\prime}} S_{k} S_{p}} \\
& \times\left\langle\rho_{\mathbf{k}^{\prime}}^{*} \rho_{\mathbf{p}^{\prime}}^{*} e_{-}^{\int_{t_{1}}^{t} d s Q \Omega^{\dagger}(s) Q} \rho_{\mathbf{k}} \rho_{\mathbf{p}}\right\rangle
\end{aligned}
$$

where the vertex functions are given by

$$
\begin{gathered}
V_{\mathbf{k}^{\prime} \mathbf{p}^{\prime}}^{(1)}=\left\langle\boldsymbol{\kappa}\left(t_{1}\right): \hat{\boldsymbol{\sigma}} Q \rho_{\mathbf{k}^{\prime}} \rho_{\mathbf{p}^{\prime}}\right\rangle \\
V_{\mathbf{k p}}^{(2)}=\left\langle\rho_{\mathbf{k}}^{*} \rho_{\mathbf{p}}^{*} Q \hat{\boldsymbol{\sigma}}\right\rangle .
\end{gathered}
$$

The second vertex is a tensor, whereas the first is a scalar because of the contraction with the velocity gradient. A straightforward calculation enables the vertices to be expressed in terms of the equilibrium static structure factor

$$
\begin{gathered}
V_{\mathbf{k}^{\prime} \mathbf{p}^{\prime}}^{(1)}=\boldsymbol{\kappa}\left(t_{1}\right): \mathbf{k}^{\prime} \mathbf{p}^{\prime} \frac{1}{k} \frac{d S_{k^{\prime}}}{d k^{\prime}} \delta_{\mathbf{k}^{\prime},-\mathbf{p}^{\prime}} \\
V_{\mathbf{k}^{\prime} \mathbf{p}^{\prime}}^{(2)}=\mathbf{k} \mathbf{p} \frac{1}{k} \frac{d S_{k}}{d k} \delta_{\mathbf{k},-\mathbf{p}},
\end{gathered}
$$

where kp indicates a dyadic product. The wave-vector restrictions imposed by the Kronecker $\delta$ s appearing in (63) and (64) reduce the fourfold sum in (60) to a double sum. We are, thus, led to consider the four-point correlator,

$$
\left\langle\rho_{\mathbf{k}^{\prime}}^{*} \rho_{-\mathbf{k}^{\prime}}^{*} e_{-}^{\int_{t_{1}}^{t} d s Q \Omega^{\dagger}(s) Q} \rho_{\mathbf{k}} \rho_{-\mathbf{k}}\right\rangle .
$$

In the spirit of quiescent mode-coupling theory we approximate the unknown four-point density correlator by a product of pair correlators and replace the $Q$-projected dynamics by the full dynamics

$$
\begin{aligned}
& \left\langle\rho_{\mathbf{k}^{\prime}}^{*} \rho_{-\mathbf{k}^{\prime}}^{*} e_{-}^{\int_{t_{1}}^{t} d s Q \Omega^{\dagger}(s) Q} \rho_{\mathbf{k}} \rho_{-\mathbf{k}}\right\rangle \\
& \quad \approx\left\langle\rho_{\mathbf{k}^{\prime}}^{*} e_{-}^{\int_{t_{1}}^{t} d s \Omega^{\dagger}(s)} \rho_{\mathbf{k}}\right\rangle\left\langle\rho_{\mathbf{k}^{\prime}}^{*} e_{-}^{\int_{t_{1}}^{t} d s \Omega^{\dagger}(s)} \rho_{\mathbf{k}}\right\rangle .
\end{aligned}
$$

For quiescent states, this approximate step is well established and amounts to assuming that the density fluctuations are Gaussian random variables [33]. We assume here that this approximation remains valid under flow.

The essential observation at this point of the calculation is that the wave vectors $\mathbf{k}$ and $\mathbf{k}^{\prime}$ appearing in (60) are not independent. The static structure factor and, consequently, the vertices (63) and (64) are clearly invariant with respect to spatial translation. In order for the stress (60) to remain translationally invariant we must, therefore, impose that the mode-coupling factorization of the four-point correlator (66) also remain invariant. It is this requirement which couples the wave vectors $\mathbf{k}$ and $\mathbf{k}^{\prime}$. Shifting all particle coordinates by a constant vector and application of (24) reveals that the the right-hand side of (66) is translationally invariant only if $\mathbf{k}^{\prime}=\mathbf{k}\left(t, t^{\prime}\right)$, where the reverse-advected wave vector is given by (26). This identification leads to the appearance of $\mathbf{k}\left(t, t^{\prime}\right)$ at several places within (60) and, thus, generates a highly nonlinear functional dependence of the stress on the velocity gradient tensor.
Substitution of (63), (64), and (66) into (60), using the identification $\mathbf{k}^{\prime}=\mathbf{k}\left(t, t^{\prime}\right)$ and replacing the remaining discrete wave-vector sum by an integral, yields

$$
\begin{aligned}
\sigma(t)= & \int_{-\infty}^{t} d t^{\prime} \int \frac{d \mathbf{k}}{16 \pi^{3}}\left[\frac{\mathbf{k}\left(t, t^{\prime}\right) \cdot \boldsymbol{\kappa}\left(t^{\prime}\right) \cdot \mathbf{k}\left(t, t^{\prime}\right)}{k k\left(t, t^{\prime}\right)}\right] \\
& \times \mathbf{k} \mathbf{k} \frac{S_{k}^{\prime} S_{k\left(t, t^{\prime}\right)}^{\prime}}{S_{k}^{2}} \Phi_{\mathbf{k}\left(t, t^{\prime}\right)}^{2}\left(t, t^{\prime}\right),
\end{aligned}
$$

where we have defined the transient density correlator

$$
\Phi_{\mathbf{k}}\left(t, t^{\prime}\right)=\frac{1}{N S_{k}}\left\langle\rho_{\mathbf{k}}^{*} e_{-}^{\int_{t^{\prime}}^{t} d s \Omega^{\dagger}(s)} \rho_{\overline{\mathbf{k}}\left(t, t^{\prime}\right)}\right\rangle,
$$

which remains to be determined. Note that the forwardadvected wave vector appears naturally in (68) as a result of

$$
\Phi_{\mathbf{k}\left(t, t^{\prime}\right)}\left(t, t^{\prime}\right)=\frac{\left\langle\rho_{\mathbf{k}\left(t, t^{\prime}\right)}^{*} e_{-}^{\int_{t^{\prime}}^{t} d s \Omega^{\dagger}(s)} \rho_{\mathbf{k}}\right\rangle}{N S_{k\left(t, t^{\prime}\right)}} .
$$

The definition in (68) will prove useful later [in Eq. (79)], when the advection of the wave vector is included into a single time evolution operator. The influence of external flow enters (67) both explicity, via the velocity gradient tensor in the prefactor, and implicitly, through the reverse-advected wave vector. The transient correlator captures the slow structural relaxation of dense suspensions, thus rendering (67) a useful tool for the study of arrested states.

Before proceeding to develop a theory for the transient correlator, it is useful to, first, rearrange (67) into an alternative form. To this end, we introduce the Finger tensor [34], a standard deformation measure from elasticity theory,

$$
\boldsymbol{B}\left(t, t^{\prime}\right)=\boldsymbol{E}\left(t, t^{\prime}\right) \boldsymbol{E}^{T}\left(t, t^{\prime}\right),
$$

where the deformation tensor is given by (32). The Finger tensor describes the stretching (but not rotation) of vectors embedded in the material. If we consider the quadratic form $\mathbf{k} \cdot \boldsymbol{B}\left(t, t^{\prime}\right) \cdot \mathbf{k}$ and take a derivative with respect to $t^{\prime}$, we obtain

$$
\begin{aligned}
\partial_{t^{\prime}}\left(\mathbf{k} \cdot \boldsymbol{B}\left(t, t^{\prime}\right) \cdot \mathbf{k}\right) & =\mathbf{k}\left(t, t^{\prime}\right) \cdot\left[\boldsymbol{\kappa}(t)+\boldsymbol{\kappa}^{T}\left(t^{\prime}\right)\right] \cdot \mathbf{k}\left(t, t^{\prime}\right) \\
& =2 \mathbf{k}\left(t, t^{\prime}\right) \cdot \boldsymbol{\kappa}(t) \cdot \mathbf{k}\left(t, t^{\prime}\right),
\end{aligned}
$$

where we have used the definition of the velocity gradient (31). Using (71) we can express our constitutive equation in the following alternative form

$$
\begin{aligned}
\boldsymbol{\sigma}(t)= & -\int_{-\infty}^{t} d t^{\prime} \int \frac{d \mathbf{k}}{32 \pi^{3}}\left[\frac{\partial}{\partial t^{\prime}}\left(\mathbf{k} \cdot \boldsymbol{B}\left(t, t^{\prime}\right) \cdot \mathbf{k}\right)\right] \\
& \times \frac{\mathbf{k} \mathbf{k}}{k}\left[\frac{S_{k}^{\prime} S_{k\left(t, t^{\prime}\right)}^{\prime}}{k\left(t, t^{\prime}\right) S_{k}^{2}}\right] \Phi_{\mathbf{k}\left(t, t^{\prime}\right)}^{2}\left(t, t^{\prime}\right) .
\end{aligned}
$$

The expression (72) provided the starting point for the development of a simpler "schematic" constitutive equation in Ref. [19].

From (72) it can be seen that the stress tensor at any given time is built by summing the contributions from all possible Fourier modes. Each of these individual contributions is obtained by integrating up the strain measure $\boldsymbol{B}\left(t, t^{\prime}\right)$ over the entire deformation history, weighted by a decaying, wavevector-dependent memory function. It is, thus, clear that strains occurring much further in the past than the longest relaxation time of $\Phi_{\mathbf{k}}\left(t, t^{\prime}\right)$ cannot contribute to the integral; such distant 
strain increments have been forgotten. Moreover, physical intuition suggests that stress contributions at differing wave vectors should be coupled, as the physical mechanisms driving relaxation on different length scales are not independent (a fact ignored in many empirical constitutive equations). Within the mode-coupling approximations to be developed below, this coupling becomes manifest in the nonlinear functional dependence of the memory function on the transient correlator; the equation of motion for $\Phi_{\mathbf{k}}\left(t, t^{\prime}\right)$ is nonlocal in $\mathbf{k}$ space.

\section{EQUATION OF MOTION}

In order to close our constitutive theory, we require an equation of motion for the transient density correlator. The fundamental approximations to be applied are very much in the spirit of standard quiescent mode-coupling theory; however, the presence of external flow necessitates considerable preparatory manipulation of formal expressions, not required in quiescent mode coupling, before the theory arrives in a form convenient for making closure approximations. The first step on this path is to identify the operators which advect the wave vector of a density fluctuation (either forwards or backward) and, thus, capture the purely affine component of the particle motion.

\section{A. Advection operators}

It is a straightforward exercise to show that a density fluctuation at advected wave vector $\overline{\mathbf{k}}\left(t, t^{\prime}\right)$ can be generated from one at $\mathbf{k}$ using the exponential advection operator

$$
e_{+}^{-\int_{t^{\prime}}^{t} d s \delta \Omega^{\dagger}(s)}\left|\rho_{\mathbf{k}}\right\rangle=\left|\rho_{\overline{\mathbf{k}}\left(t, t^{\prime}\right)}\right\rangle
$$

where the flow term in the adjoint Smoluchowski operator is given by

$$
\delta \Omega^{\dagger}(t)=\sum_{i} \mathbf{r}_{i} \cdot \boldsymbol{\kappa}^{T}(t) \cdot \boldsymbol{\partial}_{i} .
$$

The advection operator describes the purely affine time evolution of fluctuations which would occur in the absence of both Brownian motion and interparticle interactions. It is also useful to identify an analogous advection operator which acts on a density fluctuation to its left

$$
\left\langle\rho_{\mathbf{k}}^{*}\right| e_{-}^{\int_{t^{\prime}}^{t} d s \bar{\delta}^{\dagger}(s)}=\left\langle\rho_{\overline{\mathbf{k}}\left(t, t^{\prime}\right)}^{*}\right|,
$$

where the operator in the exponent is given by

$$
\overline{\delta \Omega^{\dagger}}(t)=\sum_{i} \mathbf{r}_{i} \cdot \boldsymbol{\kappa}^{T}(t) \cdot\left(\boldsymbol{\partial}_{i}+\mathbf{F}_{i}\right) .
$$

The force term $\mathbf{F}_{i}$ in (76) arises as a consequence of the equilibrium distribution function in our definition of the scalar product (48). The operations inverse to (73) and (75) which generate fluctuations at reverse-advected wave vectors are given by

$$
\begin{gathered}
e_{-}^{\int_{t^{\prime}}^{t} d s \delta \Omega^{\dagger}(s)}\left|\rho_{\mathbf{k}}\right\rangle=\left|\rho_{\mathbf{k}\left(t, t^{\prime}\right)}\right\rangle, \\
\left\langle\rho_{\mathbf{k}}^{*}\right| e_{+}^{-\int_{t^{\prime}}^{t} d s \overline{\delta \Omega}^{\dagger}(s)}=\left\langle\rho_{\mathbf{k}\left(t, t^{\prime}\right)}^{*}\right|,
\end{gathered}
$$

as can be determined from the inverse relations (A3).

\section{B. Generalized diffusion kernel}

Using (73), the transient density correlator (68) can be rewritten as

$$
\Phi_{\mathbf{k}}\left(t, t^{\prime}\right)=\frac{1}{N S_{k}}\left\langle\rho_{\mathbf{k}}^{*} U\left(t, t^{\prime}\right) \rho_{\mathbf{k}}\right\rangle,
$$

where we have defined the propagator $U\left(t, t^{\prime}\right)$ as a product of two time-evolution operators

$$
U\left(t, t^{\prime}\right) \equiv e_{-}^{\int_{t^{\prime}}^{t} d s \Omega^{\dagger}(s)} e_{+}^{-\int_{t^{\prime}}^{t} d s \delta \Omega^{\dagger}(s)} .
$$

In order to better understand the properties of $U\left(t, t^{\prime}\right)$ it is useful to express it in terms of a single exponential. Differentiation of (80) with respect to $t$ and using (A3) yields

$$
\begin{aligned}
\frac{\partial}{\partial t} U\left(t, t^{\prime}\right) & =U\left(t, t^{\prime}\right)\left[e_{-}^{\int_{t^{\prime}}^{t} d s \delta \Omega^{\dagger}(s)} \Omega_{e}^{\dagger} e_{+}^{-\int_{t^{\prime}}^{t} d s \delta \Omega^{\dagger}(s)}\right] \\
& \equiv U\left(t, t^{\prime}\right) \Omega_{\mathrm{nh}}^{\dagger}\left(t, t^{\prime}\right),
\end{aligned}
$$

where the subscript on $\Omega_{\mathrm{nh}}^{\dagger}\left(t, t^{\prime}\right)$ indicates that this operator is non-Hermitian. The formal solution of (81) is given by

$$
U\left(t, t^{\prime}\right)=e_{-}^{\int_{t^{\prime}}^{t} d s \Omega_{\mathrm{nh}}^{\dagger}\left(s, t^{\prime}\right)} .
$$

Our aim to express the transient correlator (79) in a form suitable for mode-coupling approximation is something which can be achieved only with a certain degree of foresight, combined with experience regarding the structure of the quiescent mode-coupling theory. This has the unavoidable consequence that the motivation behind some of our formal manipulations can be fully appreciated only in retrospect. We will attempt to highlight these potentially obscure steps when they occur and to motivate them as effectively as possible.

Within the quiescent mode-coupling theory the Hermiticity of the equilibrium Smoluchowski operator leads to a positive semidefinite "initial decay rate," $\Gamma_{\mathbf{k}} \equiv-\left\langle\rho_{\mathbf{k}}^{*} \Omega_{e}^{\dagger} \rho_{\mathbf{k}}\right\rangle / N S_{k}$, determining the short-time dynamics of the transient correlator [see Eq. (89) below]. Unfortunately, the operator $\Omega_{\mathrm{nh}}^{\dagger}\left(t, t^{\prime}\right)$ appearing in (82) does not retain this desirable feature and, if not treated carefully, can lead to a time-dependent initial decay rate $\Gamma_{\mathbf{k}}\left(t, t^{\prime}\right)$ which changes sign as a function of accumulated strain. The resulting zeros of $\Gamma_{\mathbf{k}}\left(t, t^{\prime}\right)$ cause divergences in the memory function $m_{\mathbf{k}}\left(t, s, t^{\prime}\right)$ [see the denominator of (103)] which destroy the numerical stability of the theory. These considerations motivate us to seek a reformulation of the propagator (80) for which the Hermitian operator

$$
\Omega_{a}^{\dagger}\left(t, t^{\prime}\right)=e_{-}^{\int_{t^{\prime}}^{t} d s \overline{\delta \Omega^{\dagger}}(s)} \Omega_{e}^{\dagger} e_{+}^{-\int_{t^{\prime}}^{t} d s \delta \Omega^{\dagger}(s)}
$$

becomes responsible for generating the initial decay rate of the theory. The operator (83) possesses the appealing feature that the average $\left\langle\rho_{\mathbf{k}}^{*} \Omega_{a}^{\dagger}\left(t, t^{\prime}\right) \rho_{\mathbf{k}}\right\rangle$ has the same form as that obtained from the equilibrium adjoint Smoluchowski operator, except that wave vectors are replaced by their advected counterparts. Positivity of the initial decay rate is, thus, ensured from the outset. 
The operator $\Omega_{a}^{\dagger}\left(t, t^{\prime}\right)$ can be brought into the game by defining the time-dependent projection operator $P\left(t, t^{\prime}\right)$ and complement $Q\left(t, t^{\prime}\right)$

$$
\begin{gathered}
P\left(t, t^{\prime}\right)=\sum_{\mathbf{k}}\left|\rho_{\overline{\mathbf{k}}\left(t, t^{\prime}\right)}\right\rangle \frac{1}{N S_{\bar{k}\left(t, t^{\prime}\right)}}\left\langle\rho_{\overline{\mathbf{k}}\left(t, t^{\prime}\right)}^{*}\right|, \\
Q\left(t, t^{\prime}\right)=1-P\left(t, t^{\prime}\right),
\end{gathered}
$$

and employing the following exact operator identity to rewrite the propagator

$$
\begin{aligned}
e^{\int_{I^{\prime}}^{t} d s \Omega_{\mathrm{nh}}^{\dagger}\left(s, t^{\prime}\right)}= & e_{-}^{\int_{t^{\prime}}^{t} d s \mathcal{G}\left(s, t^{\prime}\right)}+\int_{t^{\prime}}^{t} d s e_{-}^{\int_{l^{\prime}}^{s} d s^{\prime} \Omega_{\mathrm{nh}}^{\dagger}\left(s^{\prime}, t^{\prime}\right)} \\
& \times \mathcal{H}\left(s, t^{\prime}\right) e_{-}^{\int_{s}^{t} d s^{\prime} \mathcal{G}\left(s^{\prime}, t^{\prime}\right)},
\end{aligned}
$$

where the operators $\mathcal{G}\left(t, t^{\prime}\right)$ and $\mathcal{H}\left(t, t^{\prime}\right)$ are given by

$$
\begin{aligned}
& \mathcal{G}\left(t, t^{\prime}\right)=e_{-}^{\int_{t^{\prime}}^{t} d s \delta \Omega^{\dagger}(s)} Q\left(t, t^{\prime}\right) \Omega_{e}^{\dagger} e_{+}^{-\int_{t^{\prime}}^{t} d s \delta \Omega^{\dagger}(s)}, \\
& \mathcal{H}\left(t, t^{\prime}\right)=e_{-}^{\int_{f^{\prime}}^{t} d s \delta \Omega^{\dagger}(s)} P\left(t, t^{\prime}\right) \Omega_{e}^{\dagger} e_{+}^{-\int_{t^{\prime}}^{t} d s \delta \Omega^{\dagger}(s)} .
\end{aligned}
$$

The identity (86) may be verified by differentiation and, despite its complex structure, is simply a further time-dependent generalization of (52) employed earlier. Note that the advection operator $\overline{\delta \Omega^{\dagger}}(s)$, which is needed to build our desired Hermitian operator $\Omega_{a}^{\dagger}\left(t, t^{\prime}\right)$, enters the calculation via the advected density fluctuation on the right of the projector (84).

We next take the derivative of (86) with respect to $t$ and use the lengthy expression obtained to calculate matrix elements between density fluctuations $\left\langle\rho_{\mathbf{k}}^{*}|\cdots| \rho_{\mathbf{k}}\right\rangle / N S_{k}$. This calculation yields an equation of motion for the transient density correlator featuring a generalized diffusion kernel

$$
\begin{aligned}
& \frac{\partial}{\partial t} \Phi_{\mathbf{k}}\left(t, t^{\prime}\right)+\Gamma_{\mathbf{k}}\left(t, t^{\prime}\right) \Phi_{\mathbf{k}}\left(t, t^{\prime}\right)+\int_{t^{\prime}}^{t} d s M_{\mathbf{k}}\left(t, s, t^{\prime}\right) \Phi_{\mathbf{k}}\left(s, t^{\prime}\right) \\
& \quad=\Delta_{\mathbf{k}}\left(t, t^{\prime}\right) .
\end{aligned}
$$

Because matrix elements are taken with respect to a density fluctuation at a single wave vector, translational invariance eliminates the wave-vector sum appearing in (84).

The initial decay rate $\Gamma_{\mathbf{k}}\left(t, t^{\prime}\right)$ depends on the Hermitian operator $\Omega_{a}^{\dagger}\left(t, t^{\prime}\right)$ and can be explicitly evaluated in terms of the advected wave vector and static structure factor

$$
\Gamma_{\mathbf{k}}\left(t, t^{\prime}\right)=-\frac{\left\langle\rho_{\mathbf{k}}^{*} \Omega_{a}^{\dagger}\left(t, t^{\prime}\right) \rho_{\mathbf{k}}\right\rangle}{N S_{\bar{k}\left(t, t^{\prime}\right)}}=\frac{\bar{k}^{2}\left(t, t^{\prime}\right)}{S_{\bar{k}\left(t, t^{\prime}\right)}} .
$$

The initial decay rate takes the same functional form as in equilibrium, albeit with the static wave vector replaced by the advected one. For noninteracting particles only the first two terms in (89) survive and we obtain the exact solution for the correlator in this case, incorporating the phenomena of Taylor dispersion (enhanced diffusion in the direction of flow) [35].

The generalized diffusion kernel entering (89) is given by the formal expression

$$
M_{\mathbf{k}}\left(t, s, t^{\prime}\right)=-\frac{\left\langle B_{\mathbf{k}}^{*}\left(s, t^{\prime}\right) \tilde{U}\left(t, s, t^{\prime}\right) A_{\mathbf{k}}\left(t, t^{\prime}\right)\right\rangle}{N S_{\bar{k}\left(s, t^{\prime}\right)}},
$$

where we have defined several new operators in an effort to streamline the notation

$$
\begin{aligned}
\tilde{U}\left(t, s, t^{\prime}\right) & =e_{-}^{\int_{s}^{t} d s^{\prime} \mathcal{G}\left(s^{\prime}, t^{\prime}\right),} \\
B_{\mathbf{k}}^{*}\left(t, t^{\prime}\right) & =\rho_{\mathbf{k}}^{*} \Omega_{a}^{\dagger}\left(t, t^{\prime}\right), \\
A_{\mathbf{k}}\left(t, t^{\prime}\right) & =\mathcal{G}\left(t, t^{\prime}\right) \rho_{\mathbf{k}} .
\end{aligned}
$$

A striking feature of (91) is that the diffusion kernel is a function of three time arguments. We recall that for quiescent systems the memory function depends on only a time difference [32], whereas for systems under steady shear it depends on two time arguments [16]. The former property is a consequence of the time translational invariance of the quiescent state (ageing of arrested states being neglected within MCT), whereas the latter property is formal recognition of the existence of an absolute reference time, namely the time at which the flow was switched on. In practice, however, the residual transient may be vanishingly small for the steady states of interest. The three-time argument memory function (91) may be interpreted as describing the decay of memory between times $s$ and $t$, incorporating the coupling to the stress which is still relaxing from the strain accumulated between times $t^{\prime}$ and $t$.

The final term in the equation of motion for the transient correlator (89) is the "remainder" term on the right-hand side, given by

$$
\Delta_{\mathbf{k}}\left(t, t^{\prime}\right)=\frac{1}{N S_{k}}\left\langle\rho_{\mathbf{k}}^{*} \tilde{U}\left(t, t^{\prime}, t^{\prime}\right) \mathcal{G}\left(t, t^{\prime}\right) \rho_{\mathbf{k}}\right\rangle
$$

We know rather little about the properties of this term and the fact that it remains finite represents a primary drawback of the the present approach. (Below we will need to approximate it by zero.) Its existence stems from our use of the time-dependent projection operator (85) which only eliminates linear coupling to the density at $t=t^{\prime}$, leading to $\Delta_{\mathbf{k}}(t, t)=0$, but not at differing values of the time arguments.

\section{Generalized friction kernel}

Equation (89) represents an exact equation of motion for the transient density correlator in terms of a generalized diffusion kernel. However, experience with quiescent modecoupling theory has shown that it is difficult to accurately approximate a diffusion kernel and that far better results can be obtained when starting from an equation of motion involving a friction kernel [15]. We thus seek to convert the generalized diffusion kernel $M_{\mathbf{k}}\left(t, s, t^{\prime}\right)$ to a generalized friction kernel $m_{\mathbf{k}}\left(t, s, t^{\prime}\right)$. Following standard mode-coupling arguments, the transformation from a diffusion to a friction kernel is achieved by splitting the time-evolution operator entering the diffusion kernel, $\tilde{U}\left(t, s, t^{\prime}\right)$ in the present case, into "reducible" and "irreducible" components. As with our previous formal manipulations, foresight is required in making this step. The specific choice of how to split the time-evolution operator is made strategically, with a view to obtain an equation of the form (102) which, together with (89) can then be conveniently solved to yield (106), the desired equation of motion. 
The first step is to take the time derivative of $\tilde{U}\left(t, s, t^{\prime}\right)$. Using (92), this yields

$$
\frac{\partial}{\partial t} \tilde{U}\left(t, s, t^{\prime}\right)=\tilde{U}\left(t, s, t^{\prime}\right) \mathcal{G}\left(t, t^{\prime}\right) .
$$

We next define a non-Hermitian, time-dependent projection operator,

$$
\tilde{P}\left(t, t^{\prime}\right)=\sum_{\mathbf{k}}\left|\rho_{\mathbf{k}}\right\rangle \frac{1}{\left\langle\rho_{\mathbf{k}}^{*} \Omega_{a}^{\dagger}\left(t, t^{\prime}\right) \rho_{\mathbf{k}}\right\rangle}\left\langle\rho_{\mathbf{k}}^{*} \Omega_{a}^{\dagger}\left(t, t^{\prime}\right)\right|,
$$

with complement $\tilde{Q}\left(t, t^{\prime}\right)=1-\tilde{P}\left(t, t^{\prime}\right)$, and use this to split $\mathcal{G}\left(t, t^{\prime}\right)$ into two contributions,

$$
\begin{aligned}
\frac{\partial}{\partial t} \tilde{U}\left(t, s, t^{\prime}\right) & =\tilde{U}\left(t, s, t^{\prime}\right) \mathcal{G}\left(t, t^{\prime}\right)\left[\tilde{Q}\left(t, t^{\prime}\right)+\tilde{P}\left(t, t^{\prime}\right)\right] \\
& =\tilde{U}\left(t, s, t^{\prime}\right) \mathcal{G}^{\operatorname{irr}}\left(t, t^{\prime}\right)+\tilde{U}\left(t, s, t^{\prime}\right) \mathcal{G}^{\mathrm{red}}\left(t, t^{\prime}\right) .
\end{aligned}
$$

The irreducible operator is simply defined by $\mathcal{G}^{\text {irr }}\left(t, t^{\prime}\right)=$ $\mathcal{G}\left(t, t^{\prime}\right) \tilde{Q}\left(t, t^{\prime}\right)$, and nothing more can be done with it, whereas the reducible operator can be expressed in terms of previously encountered quantities,

$$
\mathcal{G}^{\text {red }}\left(t, t^{\prime}\right)=\left|A_{\mathbf{k}}\left(t, t^{\prime}\right)\right\rangle \frac{1}{S_{k} \Gamma_{\mathbf{k}}\left(t, t^{\prime}\right)}\left\langle B_{\mathbf{k}}^{*}\left(t, t^{\prime}\right)\right| .
$$

Treating the term $\tilde{U}\left(t, s, t^{\prime}\right) \mathcal{G}^{\text {red }}\left(t, t^{\prime}\right)$ appearing in (98) as an inhomogeneity leads to the formal solution

$$
\begin{aligned}
\tilde{U}\left(t, s, t^{\prime}\right)= & U^{\mathrm{irr}}\left(t, s, t^{\prime}\right)+\int_{s}^{t} d t^{\prime \prime} \tilde{U}\left(t^{\prime \prime}, s, t^{\prime}\right) \\
& \times \mathcal{G}^{\mathrm{red}}\left(t^{\prime \prime}, t^{\prime}\right) U^{\mathrm{irr}}\left(t, t^{\prime \prime}, t^{\prime}\right),
\end{aligned}
$$

where the irreducible time evolution is given by

$$
U^{\mathrm{irr}}\left(t, s, t^{\prime}\right)=e_{-}^{\int_{s}^{t} d s^{\prime} \mathcal{G}^{\mathrm{ir}}\left(s^{\prime}, t^{\prime}\right)},
$$

which is the solution of the corresponding homogeneous equation. Using (93) and (94) to take matrix elements of (100), $\left\langle B_{\mathbf{k}}^{*}\left(s^{\prime}, t^{\prime}\right)|\cdots| A_{\mathbf{k}}\left(t, t^{\prime}\right)\right\rangle$, and using (99) thus leads to an important equation relating the diffusion and friction kernels

$$
\begin{aligned}
M_{\mathbf{k}}\left(t, s, t^{\prime}\right)= & -\Gamma_{\mathbf{k}}\left(s, t^{\prime}\right) m_{\mathbf{k}}\left(t, s, t^{\prime}\right) \Gamma_{\mathbf{k}}\left(t, t^{\prime}\right) \\
& -\int_{s}^{t} d t^{\prime \prime} M_{\mathbf{k}}\left(t^{\prime \prime}, s, t^{\prime}\right) m_{\mathbf{k}}\left(t, t^{\prime \prime}, t^{\prime}\right) \Gamma_{\mathbf{k}}\left(t, t^{\prime}\right) .
\end{aligned}
$$

The generalized friction kernel is given by

$$
m_{\mathbf{k}}\left(t, s, t^{\prime}\right)=\frac{\left\langle B_{\mathbf{k}}^{*}\left(s, t^{\prime}\right) U^{\mathrm{irr}}\left(t, s, t^{\prime}\right) A_{\mathbf{k}}\left(t, t^{\prime}\right)\right\rangle}{N S_{\bar{k}\left(s, t^{\prime}\right)} \Gamma_{\mathbf{k}}\left(s, t^{\prime}\right) \Gamma_{\mathbf{k}}\left(t, t^{\prime}\right)},
$$

which involves the irreducible dynamics. At this stage in the calculation the importance of enforcing a positive definite initial decay rate becomes clearer. Alternative formulations for which advection can cause $\Gamma_{\mathbf{k}}\left(t, t^{\prime}\right)$ to change sign for some values of $t$ and $t^{\prime}$ will inevitably lead to undesirable singularities in the memory kernel (103). While such singularities could, in principle, be integrable and, thus, remain physical, they would at the very least prove inconvenient for numerical implementations of the theory.

We have now almost arrived at our goal to reformulate the equation of motion. The final step is to combine (89) with (102), with the former expressed as an inhomogeneous integral equation,

$$
\Phi_{\mathbf{k}}\left(t, t^{\prime}\right)=\frac{-1}{\Gamma_{\mathbf{k}}\left(t, t^{\prime}\right)} \int_{t^{\prime}}^{t} d s M_{\mathbf{k}}\left(t, s, t^{\prime}\right) \Phi_{\mathbf{k}}\left(s, t^{\prime}\right)+g_{\mathbf{k}}\left(t, t^{\prime}\right) .
$$

This equation can be viewed as a Volterra equation of the second kind [36] with an inhomogeneity given by

$$
g_{\mathbf{k}}\left(t, t^{\prime}\right)=\frac{-1}{\Gamma_{\mathbf{k}}\left(t, t^{\prime}\right)}\left[\frac{\partial}{\partial t} \Phi_{\mathbf{k}}\left(t, t^{\prime}\right)-\Delta_{\mathbf{k}}\left(t, t^{\prime}\right)\right] .
$$

The solution of this equation follows directly from the theory of Volterra equations (see Appendix D) and yields the final form for the equation of motion

$$
\begin{aligned}
& \frac{\partial}{\partial t} \Phi_{\mathbf{k}}\left(t, t^{\prime}\right)+\Gamma_{\mathbf{k}}\left(t, t^{\prime}\right)\left[\Phi_{\mathbf{k}}\left(t, t^{\prime}\right)\right. \\
& \left.\quad+\int_{t^{\prime}}^{t} d s m_{\mathbf{k}}\left(t, s, t^{\prime}\right) \frac{\partial}{\partial s} \Phi_{\mathbf{k}}\left(s, t^{\prime}\right)\right]=\tilde{\Delta}_{\mathbf{k}}\left(t, t^{\prime}\right),
\end{aligned}
$$

where the modified remainder term $\tilde{\Delta}_{\mathbf{k}}\left(t, t^{\prime}\right)$ is given in terms of previously defined quantities,

$$
\tilde{\Delta}_{\mathbf{k}}\left(t, t^{\prime}\right)=\Delta_{\mathbf{k}}\left(t, t^{\prime}\right)+\Gamma_{\mathbf{k}}\left(t, t^{\prime}\right) \int_{t^{\prime}}^{t} d s m_{\mathbf{k}}\left(t, s, t^{\prime}\right) \Delta_{\mathbf{k}}\left(s, t^{\prime}\right) .
$$

Although the integral expression (107) appears to be rather intractable, it can be simplified to a single equilibrium average. Substituting the formal solution (100) into our expression for the remainder term (95) recovers (107) with the modified remainder identified as

$$
\tilde{\Delta}_{\mathbf{k}}\left(t, t^{\prime}\right)=\frac{1}{N S_{k}}\left\langle\rho_{\mathbf{k}}^{*} U^{\mathrm{irr}}\left(t, t^{\prime}, t^{\prime}\right) \mathcal{G}\left(t, t^{\prime}\right) \rho_{\mathbf{k}}\right\rangle,
$$

by inspection. The time evolution of $\tilde{\Delta}_{\mathbf{k}}\left(t, t^{\prime}\right)$ appearing in the equation of motion for the correlator (106) is, thus, shown to be determined by the irreducible dynamics.

\section{Closure approximation}

The equation of motion (106) we have obtained is formally exact, but still contains two unknown quantities, the generalized friction kernel $m_{\mathbf{k}}\left(t, s, t^{\prime}\right)$ and the remainder term $\tilde{\Delta}_{\mathbf{k}}\left(t, t^{\prime}\right)$, both of which require approximation to arrive at a closed theory. This final stage in our development of a constitutive theory consists of two distinct steps. The first of these is specific to systems under external flow and involves the neglect of certain "strain energy" terms, which, it is hoped, are of minor importance in determining the relaxation of the transient correlator. Our choice to neglect these terms, which do not arise in quiescent mode-coupling theory, represents the only point in our development at which an approximation is made which lies beyond the established canon of mode-coupling projections and Gaussian factorizations. Once accepted, this new approximation yields directly an appealingly symmetric form for the friction kernel (103) and causes the remainder term (108) to vanish identically. The second and final step is then a rather standard projection of the friction kernel onto density pairs and factorization of the resulting four-point average. 
We consider first the remainder term (108). As described in detail in Appendix E, making the assumption that the two strain-energy terms introduced there,

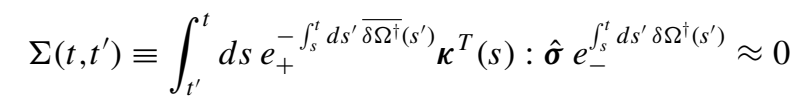

$$
\bar{\Sigma}\left(t, t^{\prime}\right) \equiv \int_{t^{\prime}}^{t} d s e_{+}^{-\int_{s}^{t} d s^{\prime} \delta \Omega^{\dagger}\left(s^{\prime}\right)} \boldsymbol{\kappa}^{T}(s): \hat{\boldsymbol{\sigma}} e_{-}^{\int_{s}^{t} d s^{\prime} \overline{\delta \Omega^{\dagger}}\left(s^{\prime}\right)} \approx 0,
$$

both vanish, gives rise to the simplifications

$$
\begin{aligned}
\mathcal{G}\left(t, t^{\prime}\right) & \approx \mathcal{G}_{Q}\left(t, t^{\prime}\right) \\
U^{\mathrm{irr}}\left(t, s, t^{\prime}\right) & \approx U_{Q}^{\mathrm{irr}}\left(t, s, t^{\prime}\right),
\end{aligned}
$$

where $\mathcal{G}_{Q}\left(t, t^{\prime}\right)$ and $U_{Q}^{\mathrm{irr}}\left(t, s, t^{\prime}\right)$ are given by (E8) and (E10), respectively. We thus find that the remainder term vanishes identically,

$$
\tilde{\Delta}_{\mathbf{k}}\left(t, t^{\prime}\right) \approx \frac{1}{N S_{k}}\left\langle\rho_{\mathbf{k}}^{*} U_{Q}^{\mathrm{irr}}\left(t, t^{\prime}, t^{\prime}\right) \mathcal{G}_{Q}\left(t, t^{\prime}\right) \rho_{\mathbf{k}}\right\rangle=0,
$$

as both operators in the average are orthogonal to linear density fluctuations.

Our assumption that the strain-energy terms (109) and (110) vanish is certainly an uncontrolled approximation, but may be partially motivated by considering their dependence on strain. For the case of steady flow [16], both $\Sigma$ terms increase in proportion to the strain accumulated since startup and thus have an influence which depends on whether the correlator has relaxed completely for small strain values. For glassy states our neglect of the $\Sigma$ terms is thus equivalent to requiring that the yield strain remain small, which is consistent with simulation results $[18,37,38]$. In any case, if the yield strain were found to be large, then the implicit assumption of a harmonic free energy functional underpinnning the entire mode-coupling approach would be invalidated, such that the new strain-energy terms would be among the least of our worries.

We turn now to the friction kernel (103). Using the previously derived relations (73), (75), (83), (87), (93), and (94) we write the numerator in the following, somewhat more explicit, form

$$
\begin{aligned}
& \left\langle B_{\mathbf{k}}^{*}\left(s, t^{\prime}\right) U^{\mathrm{irr}}\left(t, s, t^{\prime}\right) A_{\mathbf{k}}\left(t, t^{\prime}\right)\right\rangle \\
& =\left\langle\rho_{\mathbf{\mathbf { k }}\left(s, t^{\prime}\right)}^{*} \Omega_{e}^{\dagger} e_{+}^{-\int_{t^{\prime}}^{s} d s^{\prime} \delta \Omega^{\dagger}\left(s^{\prime}\right)} U^{\mathrm{irr}}\left(t, s, t^{\prime}\right) e_{-}^{\int_{t^{\prime}}^{t} d s \delta \Omega^{\dagger}(s)}\right. \\
& \left.\quad \times Q\left(t, t^{\prime}\right) \Omega_{e}^{\dagger} \rho_{\overline{\mathbf{k}}\left(t, t^{\prime}\right)}\right\rangle .
\end{aligned}
$$

Approximation (110) enables us to replace $\delta \Omega^{\dagger}(s)$ in the leftmost advection operator by $\overline{\delta \Omega^{\dagger}}(s)$ [see (E2)]

$$
e_{+}^{-\int_{t^{\prime}}^{s} d s^{\prime} \delta \Omega^{\dagger}\left(s^{\prime}\right)} \approx e_{+}^{-\int_{t^{\prime}}^{s} d s^{\prime} \delta \Omega^{\dagger}\left(s^{\prime}\right)},
$$

which, together with (112), can be used to cast the numerator of the friction kernel, Eq. (103), in the more symmetrical form,

$$
\begin{aligned}
& \left\langle\rho_{\overline{\mathbf{k}}\left(s, t^{\prime}\right)}^{*} \Omega_{e}^{\dagger} Q\left(s, t^{\prime}\right) e_{+}^{-\int_{t^{\prime}}^{s} d s^{\prime} \overline{\delta \Omega^{\dagger}\left(s^{\prime}\right)}} U_{Q}^{\mathrm{irr}}\left(t, s, t^{\prime}\right)\right. \\
& \left.\times e_{-}^{\int_{t^{\prime}}^{t} d s \delta \Omega^{\dagger}(s)} Q\left(t, t^{\prime}\right) \Omega_{e}^{\dagger} \rho_{\overline{\mathbf{k}}\left(t, t^{\prime}\right)}\right\rangle .
\end{aligned}
$$

The projected irreducible propagator $U_{Q}^{\mathrm{irr}}\left(t, s, t^{\prime}\right)$ is given by (E10) and acts in the space perpendicular to linear density fluctuations. It is this fact which enables us to insert, without incurring further approximation, the extra projector $Q\left(t, t^{\prime}\right)$ on the left of the irreducible propagator. The quantities $Q\left(t, t^{\prime}\right) \Omega_{e}^{\dagger} \rho_{\overline{\mathbf{k}}\left(t, t^{\prime}\right)}$ appearing on either side of the propagator in (116) are fluctuating forces which do not couple to linear density fluctuations.

We next introduce the time-dependent projection operator onto density pairs

$$
P_{2}\left(t, t^{\prime}\right)=\sum_{\mathbf{q}>\mathbf{p}} \frac{\left|\rho_{\overline{\mathbf{q}}\left(t, t^{\prime}\right)} \rho_{\overline{\mathbf{p}}\left(t, t^{\prime}\right)}\right\rangle\left\langle\rho_{\overline{\mathbf{q}}\left(t, t^{\prime}\right)}^{*} \rho_{\overline{\mathbf{p}}\left(t, t^{\prime}\right)}^{*}\right|}{N^{2} S_{\bar{q}\left(t, t^{\prime}\right)} S_{\bar{p}\left(t, t^{\prime}\right)}},
$$

which is simply (58) evaluated at the advected wave vector and therefore subject to the same Gaussian approximation in the denominator. The numerator (116) is thus approximated by

$$
\begin{aligned}
& \left\langle\rho_{\hat{\mathbf{k}}^{\prime}\left(s, t^{\prime}\right)}^{*} \Omega_{e}^{\dagger} Q\left(s, t^{\prime}\right) P_{2}\left(s, t^{\prime}\right) e_{+}^{-\int_{t^{\prime}}^{s} d s^{\prime} \overline{\delta \Omega^{\dagger}\left(s^{\prime}\right)}} U_{Q}^{\mathrm{irr}}\left(t, s, t^{\prime}\right)\right. \\
& \left.\times e_{-}^{\int_{t^{\prime}}^{t} d s \delta \Omega^{\dagger}(s)} P_{2}\left(t, t^{\prime}\right) Q\left(t, t^{\prime}\right) \Omega_{e}^{\dagger} \rho_{\overline{\mathbf{k}}\left(t, t^{\prime}\right)}\right\rangle, \\
& \approx \sum_{\substack{\mathbf{q}>\mathbf{p} \\
\mathbf{q}^{\prime}>\mathbf{p}^{\prime}}} \frac{V_{\mathbf{k q p}}^{(1)}\left(s, t^{\prime}\right) V_{\mathbf{k} \mathbf{q}^{\prime} \mathbf{p}^{\prime}}^{(2)}\left(t, t^{\prime}\right)}{N^{2}}\left\langle\rho_{\overline{\mathbf{q}}\left(s, t^{\prime}\right)}^{*} \rho_{\overline{\mathbf{p}}\left(s, t^{\prime}\right)}^{*}\right. \\
& \left.\times e_{+}^{-\int_{t^{\prime}}^{s} d s^{\prime} \overline{\delta \Omega^{\dagger}\left(s^{\prime}\right)}} U_{Q}^{\mathrm{irr}}\left(t, s, t^{\prime}\right) e_{-}^{\int_{t^{t}}^{t} d s \delta \Omega^{\dagger}(s)} \rho_{\overline{\mathbf{q}}^{\prime}\left(t, t^{\prime}\right)} \rho_{\overline{\mathbf{p}}^{\prime}\left(t, t^{\prime}\right)}\right\rangle,
\end{aligned}
$$

where the vertex functions are given by

$$
\begin{array}{r}
V_{\mathbf{k q p}}^{(1)}\left(s, t^{\prime}\right)=\frac{\left\langle\rho_{\overline{\mathbf{k}}\left(s, t^{\prime}\right)}^{*} \Omega_{e}^{\dagger} Q\left(s, t^{\prime}\right) \rho_{\overline{\mathbf{q}}\left(s, t^{\prime}\right)} \rho_{\overline{\mathbf{p}}\left(s, t^{\prime}\right)}\right\rangle}{N S_{\bar{q}\left(s, t^{\prime}\right)} S_{\bar{p}\left(s, t^{\prime}\right)}}, \\
V_{\mathbf{k} \mathbf{q}^{\prime} \mathbf{p}^{\prime}}^{(2)}\left(t, t^{\prime}\right)=\frac{\left\langle\rho_{\mathbf{q}^{\prime}\left(t, t^{\prime}\right)}^{*} \rho_{\mathbf{p}^{\prime}\left(t, t^{\prime}\right)}^{*} Q\left(t, t^{\prime}\right) \Omega_{e}^{\dagger} \rho_{\overline{\mathbf{k}}\left(t, t^{\prime}\right)}\right\rangle}{N S_{\bar{q}^{\prime}\left(t, t^{\prime}\right)} S_{\bar{p}^{\prime}\left(t, t^{\prime}\right)}} .
\end{array}
$$

Employing the factorization approximation for the triplet static structure factor $S_{\mathrm{kqp}}^{(3)} \approx S_{\mathbf{k}} S_{\mathbf{q}} S_{\mathbf{p}}$ enables the vertices to be expressed in terms of known two-point static correlations $V_{\mathbf{k q p}}^{(1)}\left(s, t^{\prime}\right)=\overline{\mathbf{k}}\left(s, t^{\prime}\right) \cdot\left[\overline{\mathbf{q}}\left(s, t^{\prime}\right) c_{\bar{q}\left(s, t^{\prime}\right)}+\overline{\mathbf{p}}\left(s, t^{\prime}\right) c_{\bar{p}\left(s, t^{\prime}\right)}\right] \rho \delta_{\overline{\mathbf{k}}, \overline{\mathbf{q}}+\overline{\mathbf{p}}}$,

$$
\begin{aligned}
V_{\mathbf{k q} \mathbf{q}^{\prime}}^{(2)}\left(t, t^{\prime}\right)= & \overline{\mathbf{k}}^{\prime}\left(t, t^{\prime}\right) \cdot\left[\overline{\mathbf{q}}^{\prime}\left(t, t^{\prime}\right) c_{\bar{q}^{\prime}\left(t, t^{\prime}\right)}+\overline{\mathbf{p}}^{\prime}\left(t, t^{\prime}\right) c_{\bar{p}^{\prime}\left(t, t^{\prime}\right)}\right] \\
& \times \rho \delta_{\overline{\mathbf{k}}^{\prime}, \overline{\mathbf{q}}^{\prime}+\overline{\mathbf{p}}^{\prime},},
\end{aligned}
$$

where $\rho=N / V$ is the particle number density and $c_{q}=(1-$ $\left.1 / S_{q}\right) / \rho$ is the Ornstein-Zernike direct correlation function. The four-point correlator entering (118) is approximated in the spirit of quiescent mode-coupling theory by factorizing and replacing the $Q$-projected irreducible dynamics by the full dynamics,

$$
\begin{aligned}
& \left\langle\rho_{\overline{\mathbf{q}}\left(s, t^{\prime}\right)}^{*} \rho_{\overline{\mathbf{p}}\left(s, t^{\prime}\right)}^{*} e_{+}^{-\int_{t^{\prime}}^{s} d s^{\prime} \overline{\delta \Omega^{\dagger}\left(s^{\prime}\right)}} U_{Q}^{\mathrm{irr}}\left(t, s, t^{\prime}\right) e_{-}^{\int_{t^{\prime}}^{t} d s \delta \Omega^{\dagger}(s)} \rho_{\overline{\mathbf{q}}^{\prime}\left(t, t^{\prime}\right)} \rho_{\overline{\mathbf{p}}^{\prime}\left(t, t^{\prime}\right)}\right\rangle \\
& \quad \approx N^{2} S_{\bar{q}\left(s, t^{\prime}\right)} S_{\bar{p}\left(s, t^{\prime}\right)} \Phi_{\overline{\mathbf{k}}\left(s, t^{\prime}\right)}(t, s) \Phi_{\overline{\mathbf{p}}\left(s, t^{\prime}\right)}(t, s) \delta_{\mathbf{q}, \mathbf{q}^{\prime}} \delta_{\mathbf{k}, \mathbf{k}^{\prime}}, \quad(123
\end{aligned}
$$

where the Kronecker $\delta$ functions enforce translational invariance. Using (118) together with (121), (122), and (123) to approximate the numerator in (103) and employing the explicit 
expression (90) for the initial decay rate in the denominator thus yields our final mode-coupling approximation to the memory function,

$$
\begin{aligned}
m_{\mathbf{k}}\left(t, s, t^{\prime}\right)= & \frac{\rho}{16 \pi^{3}} \int d \mathbf{q} \frac{S_{\bar{k}\left(t, t^{\prime}\right)} S_{\bar{q}\left(s, t^{\prime}\right)} S_{\bar{p}\left(s, t^{\prime}\right)}}{\bar{k}^{2}\left(s, t^{\prime}\right) \bar{k}^{2}\left(t, t^{\prime}\right)} \\
& \times V_{\mathbf{k q p}}\left(s, t^{\prime}\right) V_{\mathbf{k q p}}\left(t, t^{\prime}\right) \Phi_{\overline{\mathbf{q}}\left(s, t^{\prime}\right)}(t, s) \Phi_{\overline{\mathbf{p}}\left(s, t^{\prime}\right)}(t, s) .
\end{aligned}
$$

The wave-vector restrictions enforced by the Kronecker $\delta$ functions appearing in both the vertices and the mode-coupling factorization of the four-point correlation function reduce the fourfold sum in (118) to a single sum over $\mathbf{q}$ [which in (124) has been replaced by an integral] and leads to a coupling of the two "internal" wave vectors $\mathbf{q}$ and $\mathbf{p}$ via the condition $\mathbf{p}=\mathbf{q}-\mathbf{k}$.

We have thus arrived at a closed constitutive equation for the rheology of colloidal suspensions which requires only the number density $\rho$, the static structure factor $S_{q}$, and velocity gradient tensor $\kappa(t)$ as input. In order to calculate the stress tensor one must first solve the nonlinear integrodifferential equation (106) for the transient density correlator using the approximation $\tilde{\Delta}_{\mathbf{k}}\left(t, t^{\prime}\right)=0$ and the approximate memory function (124) with vertex functions (121) and (122). The choice of flow enters via the advected wave vectors. The transient correlator thus obtained is then substituted into the constitutive equation (72) and integrated. There are no adjustable parameters in our theory as such, although we accept that the approximations we have made are not the only ones possible. (In that sense, our theory is one of a larger family that could be specified by adjusting parameters that remain, at this stage, unidentified.) In the absence of flow the theory for the transient correlator reduces to the familiar quiescent mode-coupling theory [32].

\section{E. Linear response}

It is informative to consider the slow-flow limit of the constitutive equation (72) for which the stress response becomes linear in the velocity gradient tensor. To obtain the linear response expression we, first, neglect the strain dependence of the transient correlator and replace advected wave vectors by their nonadvected counterparts, wherever these appear explicitly. We thus obtain

$$
\boldsymbol{\sigma}(t)=-\int_{-\infty}^{t} d t^{\prime} \int \frac{\mathbf{k} \mathbf{k} d \mathbf{k}}{32 \pi^{3}} \mathbf{k} \cdot \frac{\partial \boldsymbol{B}\left(t, t^{\prime}\right)}{\partial t^{\prime}} \cdot \mathbf{k}\left[\frac{S_{k}^{\prime} \Phi_{k}^{2}\left(t-t^{\prime}\right)}{k S_{k}}\right]^{2},
$$

where the quiescent correlator depends only on a time difference. We next expand the Finger tensor to linear order in the velocity gradient tensor,

$$
\boldsymbol{B}\left(t, t^{\prime}\right)=e_{+}^{\int_{t^{\prime}}^{t} d s \kappa(s)} e_{-}^{\int_{t^{t}}^{t} d s \kappa^{T}(s)} \approx \mathbf{1}+2 \int_{t^{\prime}}^{t} d s \overline{\boldsymbol{\kappa}}(s)
$$

where $\bar{\kappa}=\left(\boldsymbol{\kappa}+\boldsymbol{\kappa}^{T}\right) / 2$ is the symmetrized velocity gradient tensor. Substitution of (126) into (125) thus yields the desired linear response result,

$$
\boldsymbol{\sigma}^{1}(t)=\int_{-\infty}^{t} d t^{\prime} \int d \mathbf{k}\left\{\left(\mathbf{k} \cdot \overline{\boldsymbol{\kappa}}\left(t^{\prime}\right) \cdot \mathbf{k}\right) \mathbf{k} \mathbf{k}\right\}\left[\frac{S_{k}^{\prime} \Phi_{k}\left(t-t^{\prime}\right)}{16 \pi^{3} k S_{k}}\right] .
$$

The required quiescent correlator is obtained from solution of the $\kappa(t) \rightarrow 0$ limit of the equation of motion (106)

$$
\frac{\partial}{\partial t} \Phi_{k}(t)+\Gamma_{k}\left[\Phi_{k}(t)+\int_{0}^{t} d s m_{k}(t-s) \frac{\partial}{\partial s} \Phi_{k}(s)\right]=0,
$$

with initial decay rate $\Gamma_{k}=k^{2} / S_{k}$ and memory function

$$
m_{k}(t)=\rho \int d \mathbf{q} \frac{S_{k} S_{q} S_{p}}{16 \pi^{3} k^{4}} V_{\mathbf{k q p}}^{2} \Phi_{q}(t) \Phi_{p}(t),
$$

where the vertex function is given by $V_{\mathbf{k q p}}=\mathbf{k} \cdot\left(\mathbf{q} c_{q}+\mathbf{p} c_{p}\right)$.

The anisotropic part of the integrand appearing in (127) is contained within the factor $\{\cdot\}$. The angular $\mathbf{k}$ integrals can, therefore, easily be performed for a given $\kappa(t)$ and it follows that for a given steady flow rate $\dot{\gamma}$ the Trouton ratio of extensional viscosity $\eta_{e} \equiv\left(\sigma_{x x}-\sigma_{y y}\right) / \dot{\gamma}$ to shear viscosity $\eta_{s} \equiv \sigma_{x y} / \dot{\gamma}$ takes the values 4 and 3 when the extensional flow is planar and uniaxial, respectively. These simple geometrical ratios are in compliance with Trouton's rules, familiar to continuum rheologists.

The elastic limit can be accessed by partial integration of (127) and yields Hooke's law for an incompressible isotropic elastic body,

$$
\sigma(t)=2 G_{\infty} \boldsymbol{\epsilon}(t)
$$

The infinitesimal accumulated strain is given by

$$
\boldsymbol{\epsilon}(t)=\int_{-\infty}^{t} d s \bar{\kappa}(s)
$$

and the single elastic constant predicted by the theory is given by the standard mode-coupling expression for the plateau value of the shear modulus [2]

$$
G_{\infty}=\frac{1}{60 \pi^{2}} \int d \mathbf{k} k^{4}\left(\frac{S_{k}^{\prime}}{S_{k}}\right)^{2} \Phi_{k}^{2}(t \rightarrow \infty)
$$

For arrested states we recall that the long time limit of the correlator $\Phi_{k}(t \rightarrow \infty)$ remains finite and serves as an order parameter for the transition from a fluid to a glass or gel. We note that the present theory makes no prediction for the bulk modulus, as the assumed compressible flow satisfies $\operatorname{Tr} \varepsilon=0$.

\section{F. Material objectivity}

The "principle of material objectivity" expresses the requirement that the constitutive relationship between stress and strain tensors should be invariant with respect to rotation of either the material body or the observer, thus preventing an unphysical dependence of the stress on the state of rotation. Material objectivity is an approximate symmetry, based on the neglect of inertial effects on the microscopic level (i.e., the influence of centrifugal and coriolis forces on particle trajectories). Nevertheless, many soft materials display this symmetry to an excellent level of approximation. The overdamped Smoluchowski dynamics underlying our treatment excludes inertial effects from the outset and, providing that 
our approximations preserve this, our set of equations (72), (106), and (124) should be material objective.

Material objectivity can be explicity confirmed by using (26) and (27) to eliminate the advected wave vectors in favor of the deformation tensors $\boldsymbol{E}\left(t, t^{\prime}\right)$ and $\boldsymbol{B}\left(t, t^{\prime}\right)$. When the system is subject to a time-dependent rotation $\boldsymbol{R}(t)$ the deformation gradient and Finger tensors transform as [25]

$$
\begin{aligned}
& \hat{\boldsymbol{E}}\left(t, t^{\prime}\right)=\boldsymbol{R}(t) \boldsymbol{E}\left(t, t^{\prime}\right) \boldsymbol{R}^{T}\left(t^{\prime}\right), \\
& \hat{\boldsymbol{B}}\left(t, t^{\prime}\right)=\boldsymbol{R}(t) \boldsymbol{B}\left(t, t^{\prime}\right) \boldsymbol{R}^{T}(t),
\end{aligned}
$$

where the hat denotes a tensor in the rotated frame. Material objectivity is verified if the rotated stress tensor is found to be given by

$$
\hat{\boldsymbol{\sigma}}(t)=\boldsymbol{R}(t) \boldsymbol{\sigma}(t) \boldsymbol{R}^{T}(t) .
$$

A rather tedious but straightforward calculation shows that insertion of the rotated deformation tensors into our constitutive equation indeed leads to the relation (134).

\section{DISTORTED STRUCTURE FACTOR}

In order to calculate the distorted structure factor $S_{\mathbf{k}}(t)$ we use the general integration-through-transients formula with $Q$-projected dynamics (54) to calculate the average of a normalized product of two density fluctuations,

$$
\begin{aligned}
& S_{\mathbf{k}}(t)=\frac{1}{N}\left\langle\rho_{\mathbf{k}}^{*} \rho_{\mathbf{k}}\right\rangle \\
& +\frac{1}{N} \int_{-\infty}^{t} d t^{\prime}\left\langle\boldsymbol{\kappa}\left(t^{\prime}\right): \hat{\boldsymbol{\sigma}} Q e_{-}^{\int_{t^{\prime}}^{t} d s} Q \Omega^{\dagger}(s) Q Q \rho_{\mathbf{k}}^{*} \rho_{\mathbf{k}}\right\rangle .
\end{aligned}
$$

In order to arrive at a closed expression for $S_{\mathbf{k}}$, we approximate the average in the integrand using the time-dependent projection operator (117)

$$
\begin{aligned}
& \left\langle\boldsymbol{\kappa}\left(t^{\prime}\right): \hat{\boldsymbol{\sigma}} Q e_{-}^{\int_{t^{\prime}}^{t} d s Q \Omega^{\dagger}(s) Q} Q \rho_{\mathbf{k}}^{*} \rho_{\mathbf{k}}\right\rangle \\
& \approx\left\langle\kappa\left(t^{\prime}\right): \hat{\sigma} Q P_{2}\left(t, t^{\prime}\right) e_{-}^{\int_{t^{\prime}}^{t} d s Q \Omega^{\dagger}(s) Q} P_{2}\left(t, t^{\prime}\right) Q \rho_{\mathbf{k}}^{*} \rho_{\mathbf{k}}\right\rangle .
\end{aligned}
$$

The vertex function appearing to the left of the propagator is given by (61), albeit with advected wave vectors replacing static ones, whereas the vertex appearing on the right consists of two terms. The first of these terms is identical to (62), again with advected wave vectors replacing their static counterparts, whereas the second term is an isotropic contribution which we choose to neglect. Our choice to ignore this term is primarily motivated by the fact that it seems to be quantitatively small in comparison with the other terms [16]. (We note, however, that the relative smallness of the isotropic term has been judged on the basis of calculations performed within the grand-canonical ensemble [39] which are not fully consistent with the requirement of fixed $N$ imposed by the Smoluchowski equation.) If we nevertheless choose to ignore this additional isotropic contribution and perform a factorization of the dynamical four-point correlation function, we arrive at the result

$$
\begin{aligned}
S_{\mathbf{k}}(t)= & S_{k}+\int_{-\infty}^{t} d t^{\prime}\left[\frac{\mathbf{k}\left(t, t^{\prime}\right) \cdot \boldsymbol{\kappa}\left(t^{\prime}\right) \cdot \mathbf{k}\left(t, t^{\prime}\right)}{k\left(t, t^{\prime}\right)}\right] \\
& \times S_{k\left(t, t^{\prime}\right)}^{\prime} \Phi_{\mathbf{k}\left(t, t^{\prime}\right)}^{2}\left(t, t^{\prime}\right) .
\end{aligned}
$$

Simple application of the chain-rule enables us to then further simplify this expression to arrive at the final form,

$$
S_{\mathbf{k}}(t ; \kappa)=S_{k}-\int_{-\infty}^{t} d t^{\prime} \frac{\partial S_{k\left(t, t^{\prime}\right)}}{\partial t^{\prime}} \Phi_{\mathbf{k}\left(t, t^{\prime}\right)}^{2}\left(t, t^{\prime}\right) .
$$

This result has the appealing interpretation that in order to calculate the nonequilibrium flow-distorted structure factor, one has to simply integrate the affinely advected equilibrium static structure factor over the flow history, weighted by the transient correlator encoding the structural relaxation of the system. We should also note that (138) makes clear the difference in philosophy between our approach and that of Miyazaki et al. [37]. In Ref. [37] the distorted stucture factor is employed as an input to the theory. In contrast, we input the equilibrium static structure factor, which serves as proxy for the potential interactions and generate the structural distortion as an output.

A notable aspect of our result (138) is that we can make a direct connection between the distorted structure factor and our constitutive equation (72). By inspection, we find that substitution of (138) into the expression

$$
\boldsymbol{\sigma}(t)=-\frac{\rho}{16 \pi^{3}} \int d \mathbf{k} \frac{\mathbf{k} \mathbf{k}}{k} c_{k}^{\prime} S_{\mathbf{k}}(t)
$$

recovers (72) exactly. For the case of shear flow, (139) coincides with a result of Frerickson and Larson for copolymers [40], in some sense reflecting the Gaussian statistics underlying both approaches.

From (139) it can be seen that our choice to ignore the isotropic term appearing in (138) corresponds to neglecting an isotropic contribution to the stress tensor. While the uncertainty regarding this term thus prevents us from predicting with confidence the system pressure, it has no consequence with regards to the of-diagonal stress tensor elements (shear stresses) or the normal stress differences which are, after all, the quantities of most direct rheological significance. Moreover, for incompressible systems the yielding behavior of arrested states, as described by the yield stress surface [19], is invariant with respect to isotropic pressure. The nonequilibrium pressure becomes relevant when addressing flow-induced particle migration and shear banding [41]. Finally, we note that the known ensemble dependence of the linear-response Green-Kubo result for the bulk viscosity of compressible fluids [42] provides circumstantial evidence supporting our choice to suppress the isotropic contribution to (138). It seems likely that the choice of ensemble will be important when employing Green-Kubo-type formulas to calculate diagonal elements of the stress tensor.

\section{DISCUSSION}

We have presented a detailed derivation of our recently proposed constitutive equation for the rheology of dense colloidal suspensions [4]. Our theory captures the slow structural relaxation arising from potential interactions (the "cage effect") by encoding these in the decay of the transient density correlator $\Phi_{\mathbf{k}}(t)$. External flow leads to the affine advection of density fluctuations which competes with particle caging and tends to accelerate the loss of memory. It is this competition of time scales which is ultimately responsible for 
the observed transition between a shear thinning viscoelastic fluid and a yielding glass as a function of the coupling strength. The integration-through-transients formalism presented here provides a means to calculate stationary averages, correlations, and response functions $[16,43]$ under arbitrary time-dependent flow.

Our consitutive equation (72) has been simplified in Ref. [19] to a schematic level and solution of the resulting equation for a number of special cases has proved rather successful in capturing qualitative aspects of the stress response in a robust fashion. So far, schematic calculations have been performed for steady flows [19], oscillatory shear [31], step strains $[19,44]$, and superposed shear and extensional flows [45]. Of particular note is that the schematic constitutive equation predicts a dynamic yield stress surface of von Mises form [46] together with small corrections related to the first normal stress difference. If the schematic model is indeed a faithful representative of the phenomenology presented by the full microscopic expression (72), as currently seems to be the case, then these findings suggest that (72) provides a possible route to calculating the yield stress surface from first-principles theory. In particular, the details of the yield surface could then be related directly to the potential interactions between the particles.

A clear drawback of the present approach is the neglect of hydrodynamic interactions between the colloids. To a certain extent, this may be defensible within the range of flow rates for which the basic assumptions of the theory remain valid, namely Peclet numbers $\mathrm{Pe} \equiv \dot{\gamma} d^{2} / D_{0}$ less than around unity. Within this regime a simple modification of the bare diffusion coefficient may restore much of the quantitative error displayed by our Brownian dynamics-based expressions when attempting to compare with experiment. However, such superficial incorporation of hydrodynamics will not affect the yield stress values predicted by the theory (occurring in the limit $\dot{\gamma} \rightarrow 0$ ) and may, thus, provide an incomplete picture. A more comprehensive method by which hydrodynamics can be included into mode-coupling-type theories is thus desirable (perhaps along the lines of Refs. [47,48]), but care should be taken that modifications of the theory do not destroy the successful description of the hard-sphere glass transition.

Possibly the most questionable aspect of the present closure approximation is our reliance on the approximation $\Sigma=\bar{\Sigma}=$ 0 (109) and (110), which constitutes a neglect of certain stress-induced couplings, to arrive at a closed equation of motion for the transient density correlator. Other than the fact that they increase linearly with the accumulated strain, we know rather little about these flow-induced terms and one cannot draw on experience from the quiescent theory to assess their importance. The central nature of such an uncontrolled approximation within the present formulation could serve as motivation to seek an alternative formalism in which they do not occur [perhaps employing an operator other than (83).] In any case, if the yield strain were found to be large, then the implicit assumption of a harmonic free energy functional, which underpins the entire mode-coupling approach, would need correction by higher-order terms; it seems reasonable to hope that $\tilde{\Delta}_{\mathbf{k}}\left(t, t^{\prime}\right)$ can be neglected on the same basis. Some further arguments for neglecting $\tilde{\Delta}_{\mathbf{k}}\left(t, t^{\prime}\right)$, made in the context of steady shear flows but not specific to that case, are made in Ref. [16]. However, further work to understand the origin of this term and, if possible, to better justify its elimination would certainly be desirable.

\section{ACKNOWLEDGMENTS}

We thank T. Voigtmann and R. G. Larson for stimulating discussions. Work was funded in part by EPSRC EP/E030173 and by the Deutsche Forschungsgemeinschaft in Transregio TR6. J.M.B. acknowledges the support of the Swiss National Science Foundation. M.E.C. acknowledges support from the Royal Society.

\section{APPENDIX A: TIME-ORDERED EXPONENTIALS}

The positively time-ordered exponential function of an arbitrary time-dependent operator $A(t)$ is defined by the series expansion $[49,50]$

$$
\begin{aligned}
e_{+}^{\int_{t_{1}}^{t_{2}} d s A(s)}= & 1+\int_{t_{1}}^{t_{2}} d s_{1} A\left(s_{1}\right)+\int_{t_{1}}^{t_{2}} d s_{1} \int_{t_{1}}^{s_{1}} d s_{2} A\left(s_{1}\right) A\left(s_{2}\right) \\
& +\int_{t_{1}}^{t_{2}} d s_{1} \int_{t_{1}}^{s_{1}} d s_{2} \int_{t_{1}}^{s_{3}} d s_{3} A\left(s_{1}\right) A\left(s_{2}\right) A\left(s_{3}\right)+\cdots .
\end{aligned}
$$

The negatively ordered exponential is similarly defined by

$$
\begin{aligned}
e_{-}^{\int_{t_{1}}^{t_{2}} d s A(s)}= & 1+\int_{t_{1}}^{t_{2}} d s_{1} A\left(s_{1}\right)+\int_{t_{1}}^{t_{2}} d s_{1} \int_{t_{1}}^{s_{1}} d s_{2} A\left(s_{2}\right) A\left(s_{1}\right) \\
& +\int_{t_{1}}^{t_{2}} d s_{1} \int_{t_{1}}^{s_{1}} d s_{2} \int_{t_{1}}^{s_{3}} d s_{3} A\left(s_{3}\right) A\left(s_{2}\right) A\left(s_{1}\right)+\cdots .
\end{aligned}
$$

By multiplying out the series expansions in (A1) and (A2) the following identities can be proven

$$
\begin{aligned}
e_{+}^{ \pm \int_{t_{1}}^{t_{2}} d s A(s)} e_{-}^{\mp \int_{t_{1}}^{t_{2}} d s A(s)} & =1, \\
e_{-}^{ \pm \int_{t_{1}}^{t_{2}} d s A(s)} e_{+}^{\mp \int_{t_{1}}^{t_{2}} d s A(s)} & =1,
\end{aligned}
$$

where care must be taken that causality is respected within the multiple integrals. These results are consistent with the expressions (32) and (33) for the deformation gradient and inverse, respectively. Using the series expansions (A1) and (A2) the following useful results for the derivatives of timeordered exponential functions are easily proven

$$
\begin{aligned}
\frac{\partial}{\partial t_{2}}\left[e_{+}^{\int_{t_{1}}^{t_{2}} d s A(s)}\right] & =A\left(t_{2}\right)\left[e_{+}^{\int_{t_{1}}^{t_{2}} d s A(s)}\right], \\
\frac{\partial}{\partial t_{1}}\left[e_{+}^{\int_{t_{2}}^{t_{2}} d s A(s)}\right] & =-\left[e_{+}^{\int_{t_{1}}^{t_{2}} d s A(s)}\right] A\left(t_{1}\right), \\
\frac{\partial}{\partial t_{2}}\left[e_{-}^{\int_{t_{1}}^{t_{2}} d s A(s)}\right] & =\left[e_{-}^{\int_{t_{1}}^{t_{2}} d s A(s)}\right] A\left(t_{2}\right), \\
\frac{\partial}{\partial t_{1}}\left[e_{-}^{\int_{t_{1}}^{t_{2}} d s A(s)}\right] & =-A\left(t_{1}\right)\left[e_{-}^{\int_{t_{1}}^{t_{2}} d s A(s)}\right] .
\end{aligned}
$$

Finally, we note that the adjoint operation satisfies

$$
\begin{aligned}
& {\left[e_{+}^{\int_{t_{1}}^{t_{2}} d s A(s)}\right]^{\dagger}=e_{-}^{\int_{t_{1}}^{t_{2}} d s A^{\dagger}(s)},} \\
& {\left[e_{-}^{\int_{t_{1}}^{t_{2}} d s A(s)}\right]^{\dagger}=e_{+}^{\int_{t_{1}}^{t_{2}} d s A^{\dagger}(s)} .}
\end{aligned}
$$




\section{APPENDIX B: OPERATOR IDENTITY}

The following operator identity has proved useful in analyzing the translational invariance of the two-time correlation functions:

$$
\begin{aligned}
e_{-}^{\int_{t_{1}}^{t_{2}} d s[A(s)+B(s)]} & \\
= & \exp _{-}\left[\int_{t_{1}}^{t_{2}} d s A(s)\right] \\
& \times \exp _{-}\left[\int_{t_{1}}^{t_{2}} d s e_{+}^{-\int_{s}^{t_{2}} d s^{\prime} A\left(s^{\prime}\right)} B(s) e_{-}^{\int_{s}^{t_{2}} d s^{\prime} A\left(s^{\prime}\right)}\right],
\end{aligned}
$$

where $A(t)$ and $B(t)$ are two arbitrary time-dependent operators. The proof proceeds by, first, defining the operator

$$
\begin{aligned}
U^{+}\left(t_{2}, t_{1}\right)= & e_{-}^{\int_{t_{1}}^{t_{2}} d s[A(s)+B(s)]} \\
= & \sum_{n=0}^{\infty} \int_{t_{1}}^{t_{2}} d s_{1} \cdots \int_{t_{1}}^{s_{n-1}} d s_{n}\left[A\left(s_{n}\right)+B\left(s_{n}\right)\right] \\
& \times \cdots\left[A\left(s_{1}\right)+B\left(s_{1}\right)\right],
\end{aligned}
$$

where it is understood that the $n=0$ term is unity. The derivative is thus given by

$$
\frac{\partial}{\partial t_{2}} U^{+}\left(t_{2}, t_{1}\right)=U^{+}\left(t_{2}, t_{1}\right)\left[A\left(t_{2}\right)+B\left(t_{2}\right)\right] .
$$

We now define a new operator,

$$
\begin{aligned}
\tilde{U}\left(t_{2}, t_{1}\right) & =e_{-}^{\int_{t_{1}}^{t_{2}} d s c\left(t_{2}, s\right)} \\
& =\sum_{n=0}^{\infty} \int_{t_{1}}^{t_{2}} d s_{1} \cdots \int_{t_{1}}^{s_{n-1}} d s_{n} c\left(t_{2}, s_{n}\right) \cdots c\left(t_{2}, s_{1}\right),
\end{aligned}
$$

where $c\left(t_{2}, s\right)$ is at present an arbitrary function. The derivative of this new operator is given by

$$
\begin{aligned}
\frac{\partial}{\partial t_{2}} \tilde{U}\left(t_{2}, t_{1}\right)= & \tilde{U}\left(t_{2}, t_{1}\right) c\left(t_{2}, t_{2}\right) \\
& +\sum_{n=0}^{\infty} \int_{t_{1}}^{t_{2}} d s_{1} \cdots \int_{t_{1}}^{s_{n-1}} d s_{n}\left[c^{\prime}\left(t_{2}, s_{n}\right) \cdots c\left(t_{2}, s_{1}\right)\right. \\
& +c\left(t_{2}, s_{n}\right) c^{\prime}\left(t_{2}, s_{n-1}\right) \cdots c\left(t_{2}, s_{1}\right) \\
& +\cdots c\left(t_{2}, s_{n}\right) \cdots c^{\prime}\left(t_{2}, s_{2}\right) c\left(t_{2}, s_{1}\right) \\
& \left.+c\left(t_{2}, s_{n}\right) \cdots c^{\prime}\left(t_{2}, s_{1}\right)\right] .
\end{aligned}
$$

Defining $c\left(t_{2}, s\right)$ (up to boundary conditions) in the following way,

$$
\frac{\partial}{\partial t_{2}} c\left(t_{2}, s\right)=c\left(t_{2}, s\right) A\left(t_{2}\right)-A\left(t_{2}\right) c\left(t_{2}, s\right),
$$

leads to many convenient cancellations when substituted into (B5), resulting in

$$
\begin{aligned}
\frac{\partial}{\partial t_{2}} \tilde{U}\left(t_{2}, t_{1}\right)= & \tilde{U}\left(t_{2}, t_{1}\right) c\left(t_{2}, t_{2}\right) \\
& +\tilde{U}\left(t_{2}, t_{1}\right) A\left(t_{2}\right)-A\left(t_{2}\right) \tilde{U}\left(t_{2}, t_{1}\right) .
\end{aligned}
$$

Choosing the boundary condition $c\left(t_{2}, t_{2}\right)=B\left(t_{2}\right)$ yields

$$
\frac{\partial}{\partial t_{2}} \tilde{U}\left(t_{2}, t_{1}\right)+A\left(t_{2}\right) \tilde{U}\left(t_{2}, t_{1}\right)=\tilde{U}\left(t_{2}, t_{1}\right)\left(A\left(t_{2}\right)+B\left(t_{2}\right)\right) .
$$

Multiplying (B8) on the right with a negatively ordered exponential yields

$$
\frac{\partial}{\partial t_{2}}\left[e_{-}^{\int_{t_{2}}^{t_{2}} d s A(s)} \tilde{U}\left(t_{2}, t_{1}\right)\right]=\left[e_{-}^{\int_{t_{1}}^{t_{2}} d s A(s)} \tilde{U}\left(t_{2}, t_{1}\right)\right]\left[A\left(t_{2}\right)+B\left(t_{2}\right)\right] .
$$

Comparison of (B3) with (B10) allows the identification

$$
U^{+}\left(t_{2}, t_{1}\right)=e_{-}^{\int_{t_{1}}^{t_{2}} d s A(s)} \tilde{U}\left(t_{2}, t_{1}\right)
$$

Using (B2) and (B4) to write $U^{+}$and $\tilde{U}$ as exponentials thus yields

$$
e_{-}^{\int_{t_{1}}^{t_{2}} d s[A(s)+B(s)]}=e_{-}^{\int_{t_{1}}^{t_{2}} d s A(s)} e_{-}^{\int_{t_{1}}^{t_{2}} d s c\left(t_{2}, s\right)} .
$$

Finally, we require an explicit form for $c\left(t_{2}, s\right)$. Using our chosen boundary condition $c\left(t_{2}, t_{2}\right)=B\left(t_{2}\right)$ enables solution of (B6)

$$
c\left(t_{2}, s\right)=e_{+}^{-\int_{t_{1}}^{t_{2}} d s^{\prime} A\left(s^{\prime}\right)} B(s) e_{-}^{\int_{t_{1}}^{t_{2}} d s^{\prime} A\left(s^{\prime}\right)},
$$

as can be verified by substitution of (B12) into (B6). Substitution of (B12) into (B11) thus yields the desired result (B1).

\section{APPENDIX C: HADAMARD LEMMA}

For noncommuting, time-independent operators $X$ and $Y$ there exists a well-known identity (the Hadamard lemma),

$$
\begin{aligned}
e^{X} Y e^{-X}= & Y+[X, Y]+\frac{1}{2 !}[X,[X, Y]] \\
& +\frac{1}{3 !}[X,[X,[X, Y]]]+\cdots,
\end{aligned}
$$

where $[X, Y]$ is the commutator. Proof follows from defining the function $f(s)=e^{s X} Y e^{-s X}$, Taylor expanding about $s=0$ and then setting $s=1$. (C1) can be particularly useful in cases for which the sequence of nested commutators truncates at a low order or when the infinite series can be resummed into a closed form.

When employing the operator identity (B1) a structure analogous to the left-hand side of $(\mathrm{C} 1)$ arises naturally in one of the factorized exponential functions. We are, thus, motivated to find a continuous version of the Hadamard lemma, which may be useful in simplifying (B1) for certain special cases. For arbitrary time-dependent operators $A(t)$ and $B(t)$, we find the following nested commutator expansion,

$$
\begin{aligned}
e_{+}^{-\int_{s}^{t} d s^{\prime} A\left(s^{\prime}\right)} B(s) e_{-}^{\int_{s}^{t} d s^{\prime} A\left(s^{\prime}\right)} & \\
= & B(s)-\int_{s}^{t} d s_{1}\left[A\left(s_{1}\right), B(s)\right] \\
& +\int_{s}^{t} d s_{1} \int_{s}^{s_{1}} d s_{2}\left[A\left(s_{1}\right),\left[A\left(s_{2}\right), B(s)\right]\right] \\
& -\int_{s}^{t} d s_{1} \int_{s}^{s_{1}} d s_{2} \int_{s}^{s_{2}} d s_{3}\left[A\left(s_{1}\right),\left[A\left(s_{2}\right),\left[A\left(s_{3}\right), B(s)\right]\right]\right] \\
& +\cdots,
\end{aligned}
$$

which is a continuum version of (C1). The proof proceeds by, first, defining the operator

$$
F(t, s)=e_{+}^{-\int_{s}^{t} d s^{\prime} A\left(s^{\prime}\right)} B(s) e_{-}^{\int_{s}^{t} d s^{\prime} A\left(s^{\prime}\right)} .
$$


Differentiation with respect to $t$ leads to

$$
\frac{\partial F(t, s)}{\partial t}=-A(t) F(t, s)+F(t, s) A(t),
$$

where we have used (A5) and (A7). Integration of (C4) from $s$ to $t$ yields a recursion relation

$$
F(t, s)=B(s)-\int_{s}^{t} d s_{1}\left[A\left(s_{1}\right) F\left(s_{1}, s\right)-F\left(s_{1}, s\right) A\left(s_{1}\right)\right],
$$

where we have made the identification $F(s, s)=B(t)$. Straightforward iteration of (C5) directly generates the series (C2).

\section{APPENDIX D: VOLTERRA EQUATION}

A Volterra integral equation of the second kind for the function $\Phi_{\mathbf{k}}\left(t, t^{\prime}\right)$ can be written the following form [36]:

$$
\Phi_{\mathbf{k}}\left(t, t^{\prime}\right)=g_{\mathbf{k}}\left(t, t^{\prime}\right)+\int_{t^{\prime}}^{t} d s K_{\mathbf{k}}\left(t, s, t^{\prime}\right) \Phi_{\mathbf{k}}\left(s, t^{\prime}\right)
$$

The wave-vector subscripts are irrelevant in the formal solution of this temporal integral equation but are, nevertheless, included here to facilitate comparison with the equations appearing in the main text. Subject to certain reasonable conditions on both the kernel $K_{\mathbf{k}}\left(t, s, t^{\prime}\right)$ and inhomogeneity $g_{\mathbf{k}}\left(t, t^{\prime}\right)$, the solution is given by

$$
\Phi_{\mathbf{k}}\left(t, t^{\prime}\right)=g_{\mathbf{k}}\left(t, t^{\prime}\right)-\int_{t^{\prime}}^{t} d s R_{\mathbf{k}}\left(t, s, t^{\prime}\right) g_{\mathbf{k}}\left(s, t^{\prime}\right),
$$

where the resolvent kernel $R\left(t, s, t^{\prime}\right)$ satisfies the following linear integral equation

$$
R_{\mathbf{k}}\left(t, s, t^{\prime}\right)=-K_{\mathbf{k}}\left(t, s, t^{\prime}\right)+\int_{s}^{t} d s^{\prime} K_{\mathbf{k}}\left(s^{\prime}, s, t^{\prime}\right) R_{\mathbf{k}}\left(t, s^{\prime}, t^{\prime}\right) .
$$

These results can be used to solve directly the inhomogeneous equation (104). Comparing (104) with (D1) enables the identification

$$
K_{\mathbf{k}}\left(t, s, t^{\prime}\right)=-M_{\mathbf{k}}\left(t, s, t^{\prime}\right) / \Gamma_{\mathbf{k}}\left(t, t^{\prime}\right)
$$

Substitution of (D4) into (D3) and comparison of the resulting equation with (102) identifies the resolvent kernel

$$
R_{\mathbf{k}}\left(t, s, t^{\prime}\right)=-m_{\mathbf{k}}\left(t, s, t^{\prime}\right) \Gamma_{\mathbf{k}}\left(s, t^{\prime}\right) .
$$

Substitution of (D5) into (D2) yields the solution (106).

\section{APPENDIX E: SIMPLIFYING THE FRICTION KERNEL}

When attempting to simplify the friction kernel entering (106) it is useful to convert advection operators involving $\delta \Omega^{\dagger}(t)$ to analogous quantities involving $\overline{\delta \Omega^{\dagger}}(t)$. This can be achieved using the identities

$$
\begin{aligned}
e_{-}^{\int_{t^{\prime}}^{t} d s \delta \Omega^{\dagger}(s)} & =e_{-}^{\int_{t^{\prime}}^{t} d s \overline{\delta \Omega^{\dagger}}(s)}\left[1+\Sigma\left(t, t^{\prime}\right)\right], \\
e_{+}^{-\int_{t^{\prime}}^{t} d s \delta \Omega^{\dagger}(s)} & =\left[1+\bar{\Sigma}\left(t, t^{\prime}\right)\right] e_{+}^{-\int_{t^{\prime}}^{t} d s \overline{\delta \Omega^{\dagger}(s)},}
\end{aligned}
$$

where we have introduced the two operators

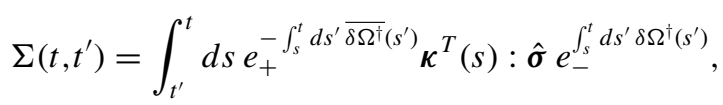

$$
\begin{aligned}
& \bar{\Sigma}\left(t, t^{\prime}\right)=\int_{t^{\prime}}^{t} d s e_{+}^{-\int_{s}^{t} d s^{\prime} \delta \Omega^{\dagger}\left(s^{\prime}\right)} \kappa^{T}(s): \hat{\sigma} e_{-}^{\int_{s}^{t} d s^{\prime} \overline{\delta \Omega^{\dagger}\left(s^{\prime}\right)}} .
\end{aligned}
$$

In the main text we will argue that both $\Sigma$ and $\bar{\Sigma}$ can be set to zero in the final approximation. Proof of (E1) follows from considering the derivative

$$
\begin{aligned}
& \frac{\partial}{\partial t^{\prime}}\left[e_{+}^{-\int_{t^{\prime}}^{t} d s \overline{\delta \Omega^{\dagger}}(s)} e_{-}^{\int_{t^{\prime}}^{t} d s \delta \Omega^{\dagger}(s)}\right]=e_{+}^{-\int_{t^{\prime}}^{t} d s \overline{\delta \Omega^{\dagger}}(s)}\left[\overline{\delta \Omega^{\dagger}}(t)-\delta \Omega^{\dagger}(t)\right] \\
& \times e_{-}^{\int_{t^{\prime}}^{t} d s \delta \Omega^{\dagger}(s)}
\end{aligned}
$$

and integration from $t^{\prime}$ to $t$. The proof of (E2) is analogous.

The identity (E1) is helpful in simplifying the irreducible time evolution operator given by

$$
\mathcal{G}^{\operatorname{irr}}\left(t, t^{\prime}\right)=\mathcal{G}\left(t, t^{\prime}\right) \tilde{Q}\left(t, t^{\prime}\right) .
$$

Using (E1) to replace the leftmost ordered exponential in (87) yields a natural division into two terms,

$$
\mathcal{G}^{\text {irr }}\left(t, t^{\prime}\right)=\left[\mathcal{G}_{Q}\left(t, t^{\prime}\right)+\mathcal{G}_{\Sigma}\left(t, t^{\prime}\right)\right] \tilde{Q}\left(t, t^{\prime}\right)
$$

where

$$
\begin{gathered}
\mathcal{G}_{Q}\left(t, t^{\prime}\right)=e_{-}^{\int_{t^{\prime}}^{t} d s \overline{\delta \Omega^{\dagger}}(s)} Q\left(t, t^{\prime}\right) \Omega_{e}^{\dagger} e_{+}^{-\int_{t^{\prime}}^{t} d s \delta \Omega^{\dagger}(s)}, \\
\mathcal{G}_{\Sigma}\left(t, t^{\prime}\right)=e_{-}^{\int_{t^{\prime}}^{t} d s \overline{\delta \Omega^{\dagger}}(s)} \Sigma\left(t, t^{\prime}\right) Q\left(t, t^{\prime}\right) \Omega_{e}^{\dagger} e_{+}^{-\int_{t^{\prime}}^{t} d s \delta \Omega^{\dagger}(s)} .
\end{gathered}
$$

The purpose of this manipulation is that under the assumption that $\Sigma\left(t, t^{\prime}\right)$ vanishes we obtain $\mathcal{G}^{\text {irr }}\left(t, t^{\prime}\right) \approx \mathcal{G}_{Q}\left(t, t^{\prime}\right) \tilde{Q}\left(t, t^{\prime}\right)$, which is an operator in the space perpendicular to linear density fluctuations. This consideration then leads us to define the propagator

$$
U_{Q}^{\mathrm{irr}}\left(t, s, t^{\prime}\right) \equiv e_{-}^{\int_{s}^{t} d s^{\prime} \mathcal{G}_{Q}\left(s^{\prime}, t^{\prime}\right) \tilde{Q}\left(s^{\prime}, t^{\prime}\right)},
$$

which generates dynamics in the space perpendicular to linear density fluctuations.
[1] J. K. G. Dhont, An Introduction to the Dynamics of Colloids (Amsterdam, Elsevier, 1996).

[2] G. Nägele and J. Bergenholtz, J. Chem. Phys. 108, 9893 (1998).

[3] J. M. Brader, J. Phys.: Condens. Matter 22, 363101 (2010).

[4] J. M. Brader, M. E. Cates, and M. Fuchs, Phys. Rev. Lett. 101, 138301 (2008).

[5] P. Coussot, Rheometry of Pastes, Suspensions, and Granualar Materials (Wiley, New York, 2005).
[6] R. G. Larson, The Structure and Rheology of Complex Fluids (Oxford University Press, Oxford, UK, 1998).

[7] J.-P. Hansen and I. R. McDonald, Theory of Simple Liquids (Academic Press, London, 1986).

[8] W. van Megen and P. N. Pusey, Phys. Rev. A 43, 5429 (1991).

[9] W. van Megen and S. M. Underwood, Phys. Rev. Lett. 70, 2766 (1993). 
[10] C. Beck, W. Härtl, and R. Hempelmann, J. Chem. Phys. 111, 8209 (1999).

[11] W. Götze, J. Phys.: Condens. Matter 11, A1 (1999).

[12] K. N. J. Pham et al., Science 296, 104 (2002).

[13] E. Zaccarelli, J. Phys.: Condens. Matter 19, 323101 (2007).

[14] M. Fuchs and M. E. Cates, Phys. Rev. Lett. 89, 248304 (2002).

[15] M. Fuchs and M. E. Cates, J. Phys.: Condens. Matter 17, S1681 (2005).

[16] M. Fuchs and M. E. Cates, J. Rheol. 53, 957 (2009).

[17] J. M. Brader, Th. Voigtmann, M. E. Cates, and M. Fuchs, Phys. Rev. Lett. 98, 058301 (2007).

[18] J. Zausch, J. Horbach, M. Laurati, S. Egelhaaf, J. M. Brader, Th. Voigtmann, and M. Fuchs, J. Phys.: Condens. Matter 20, 404210 (2008).

[19] J. M. Brader, Th. Voigtmann, M. Fuchs, R. G. Larson, and M. E. Cates, Proc. Natl. Acad. Sci. USA 106, 15186 (2009).

[20] M. Fuchs and M. E. Cates, Faraday Discuss. 123, 267 (2003).

[21] O. Henrich, F. Weysser, M. E. Cates, and M. Fuchs, Philos. Trans. R. Soc. London A 367, 5033 (2009).

[22] M. Krüger, F. Weysser, and M. Fuchs, Eur. Phys. J. E 34, 88 (2011).

[23] U. Balucani and M. Zoppi, Dynamics of the Liquid State (Oxford University Press, Oxford, UK, 1994).

[24] J. H. Irving and J. G. Kirkwood, J. Chem. Phys. 18, 817 (1950).

[25] R. G. Larson, Constitutive Equations for Polymer Melts and Solutions (Butterworth, Boston, 1988).

[26] R. B. Bird, R. C. Armstrong, and O. Hassager, Dynamics of Polymeric Liquids (Wiley, New York, 1987).

[27] D. J. Evans and G. Morriss, Statistical Mechanics of Nonequilibrium Liquids (Cambridge University Press, Cambridge, UK, 2008).

[28] J. F. Lutsko, J. Appl. Phys. 65, 2991 (1989).

[29] S.-H. Chong and B. Kim, Phys. Rev. E 79, 021203 (2009).

[30] R. Zwanzig, Nonequilibrium Statistical Mechanics (Oxford University Press, Oxford, UK, 2001).
[31] J. M. Brader, M. Siebenbürger, M. Ballauff, K. Reinheimer, M. Wilhelm, S. J. Frey, F. Weysser, and M. Fuchs, Phys. Rev. E 82, 061401 (2010).

[32] W. Götze, in Liquids, Freezing and Glass Transition (NorthHolland, Amsterdam, 1991).

[33] W. Götze and S. J. Sjögren, Rep. Prog. Phys. 55, 241 (1992).

[34] M. Doi and S. F. Edwards, The Theory of Polymer Dynamics (Oxford University Press, Oxford, UK, 1989).

[35] R. T. Foister and T. G. M. van de Ven, J. Fluid Mech. 96, 105 (1980).

[36] F. G. Tricomi, Integral Equations (Dover, London, 1985).

[37] K. Miyazaki, D. R. Reichman, and R. Yamamoto, Phys. Rev. E 70, 011501 (2004).

[38] F. Varnik, J. Chem. Phys. 125, 164514 (2006).

[39] O. Henrich, O. Pfeifroth, and M. Fuchs, J. Phys.: Condens. Matter 19, 205132 (2007).

[40] G. H. Fredrickson and R. G. Larson, J. Chem. Phys. 86, 1553 (1987).

[41] R. Besseling, L. Isa, P. Ballesta, G. Petekidis, M. E. Cates, and W. C. K. Poon, Phys. Rev. Lett. 105, 268301 (2010).

[42] R. Zwanzig, Annu. Rev. Phys. Chem. 16, 67 (1965).

[43] M. Krüger and M. Fuchs, Phys. Rev. Lett. 102, 135701 (2009).

[44] Th. Voigtmann, J. M. Brader, M. Fuchs, and M. E. Cates, Soft Matter 8, 4244 (2012).

[45] T. F. F. Farage and J. M. Brader, J. Rheol. 56, 259 (2012).

[46] R. Hill, The Mathematical Theory of Plasticity (Oxford University Press, Oxford 1971).

[47] A. J. Banchio, J. Bergenholtz, and G. Nägele, Phys. Rev. Lett. 82, 1792 (1999).

[48] A. J. Banchio, J. Bergenholtz, and G. Nägele, J. Chem. Phys. 111, 8721 (1999).

[49] N. G. van Kampen, Stochastic Processes in Physics and Chemistry (North-Holland, Amsterdam, 1981).

[50] H. Risken, The Fokker-Planck Equation (Springer, Berlin, 1996). 